\title{
WEAK NOTIONS OF JACOBIAN DETERMINANT AND RELAXATION
}

\author{
Guido De PhiLippis ${ }^{1}$
}

\begin{abstract}
In this paper we study two weak notions of Jacobian determinant for Sobolev maps, namely the distributional Jacobian and the relaxed total variation, which in general could be different. We show some cases of equality and use them to give an explicit expression for the relaxation of some polyconvex functionals.
\end{abstract}

Mathematics Subject Classification. 49J45, 28A75.

Received March 15, 2010. Revised July 15, 2010.

Published online December 2, 2010.

\section{INTRODUCTION}

The aim of this paper is to study weak notions of Jacobian determinant, $\operatorname{det} D u$, for maps $u: \Omega \subset \mathbb{R}^{n} \rightarrow \mathbb{R}^{n}$ in the Sobolev class $W^{1, p}$ for some $p$.

If $u$ is a diffeomorphism, the change of variable formula and Lebesgue differentiation Theorem give a clear geometric meaning to det $D u(x)$, it is the "infinitesimal" change of volume due to the deformation $u$. If $u$ is not a bijective map the area formulas (in the unoriented version (1.1) or in the oriented one (1.2))

$$
\begin{gathered}
\int_{\Omega}|\operatorname{det} D u(x)| \mathrm{d} x=\int_{\mathbb{R}^{n}} N(u, \Omega, y) \mathrm{d} y \\
\int_{\Omega} \operatorname{det} D u(x) \mathrm{d} x=\int_{\mathbb{R}^{n}} \operatorname{deg}(u, \Omega, y) \mathrm{d} y\left({ }^{2}\right)
\end{gathered}
$$

relate the integral of the Jacobian determinant to how many times the image of $u$ covers the target space.

If $u$ is merely a Sobolev map it is still possible to consider the area formula and the "pointwise" Jacobian $\operatorname{det} D u$ (see [26]), however if $u$ is not sufficiently regular (more precisely if $u \in W^{1, p}$ and $p<n$ ) this gives only a partial information about the behaviour of $u$.

If $p \geq n$, Hölder inequality implies $\operatorname{det} D u \in L^{\frac{p}{n}}$; moreover the map

$$
\begin{aligned}
W^{1, p} & \rightarrow L^{\frac{p}{n}} \\
u & \mapsto \operatorname{det} D u
\end{aligned}
$$

\footnotetext{
Keywords and phrases. Distributional determinant, topological degree, relaxation.

${ }^{1}$ Scuola Normale Superiore, P.za dei Cavalieri 7, 56100 Pisa, Italy. guido.dephilippis@sns.it

${ }^{2} N(u, \Omega, y)=\#\{x \in \Omega: u(x)=y\}$ is the Banach indicatrix function, while $\operatorname{deg}(u, \Omega, y)$ is the Brouwer degree of $u$ (see Sect. 2.2).
} 
is continuous if we endow both spaces with the strong topology. What is more surprising is that if $p>n$ this map is still (sequentially) continuous also if we endow the spaces with the weak topology (see Thm. 2.2). If $p=n$ we don't have continuity if we consider the $L^{1}$ weak topology for Jacobians, however we have it if we consider the weak-* topology (see for example [12], Chap. 8), however if $\operatorname{det} D u_{k} \geq 0$ we still have continuity with respect to the $L^{1}$ weak topology (see $[10,35]$ ).

This implies the semicontinuity with respect to the weak convergence of the functional:

$$
u \mapsto T V(u, \Omega):=\int_{\Omega}|\operatorname{det} D u|
$$

and, more in general, of polyconvex functionals, i.e. the ones that can be represented as the integral of a convex function of the minors of the gradient:

$$
F(u, \Omega)=\int_{\Omega} g\left(D u, \mathcal{M}_{1}(D u), \ldots, \operatorname{det} D u\right) .
$$

If $p<n$, in general det $D u$ is not a summable function; moreover also if $\operatorname{det} D u \in L^{1}$ we lose continuity and semicontinuity properties. In particular it is possible to see that:

- the function $u(x)=\frac{x}{|x|}$ is in $W^{1, p}(B)$ for any $p<n$, det $D u=0$ almost everywhere but for any sequence of smooth functions strongly converging to $u$ in $W^{1, p}(B) \cap L^{\infty}$ with $p>n-1$ we have:

$$
\operatorname{det} D u_{k} \stackrel{*}{\rightarrow} \omega_{n} \delta_{0}\left({ }^{3}\right)
$$

in the sense of distributions.

- For any smooth function $u$ there exists a sequence $\left\{u_{k}\right\} \in W^{1, p}$, with $p<n$, weakly converging to $u$ and such that det $D u_{k}=0$ almost everywhere (see Ex. 3 at p. 284 in [26] where it is shown for the map $u(x)=x$, this implies the result for any smooth function).

The main reason for this behaviour is that the pointwise Jacobian det Du doesn't count in any way the presence of fractures in the image of $u$.

We now introduce the two weak formulations we are going to study. The first one is based on the particular structure of the Jacobian determinant and it leads to the notion of distributional determinant (introduced by Ball [3] in the context of non-linear elasticity and widely studied also in different contexts, see for example $[1,9,11,14,15,28,34,36-38]$ and references therein). The second one is based on the Lebesgue-Serrin extension and it leads to the relaxed total variation (first introduced by Marcellini in [30] and then systematically studied in $[6,20-23,31-33,41])$.

Let $u$ be a $C^{2}$ map, thanks to the divergence free property of the cofactor matrix (see Sect. 2 for a precise definition)

$$
\sum_{i} \partial_{j}(\operatorname{adj} D u)_{i}^{j}=0
$$

we can express the Jacobian as a divergence

$$
\operatorname{det} D u(x)=\frac{1}{n} \sum_{i} \partial_{j}\left(\sum_{j} u^{i}(x)(\operatorname{adj} D u(x))_{i}^{j}\right)=\frac{1}{n} \operatorname{div}(\operatorname{adj} D u \cdot u) .
$$

Therefore formal integration by parts suggests that we may define the distribution:

$$
\langle J u, \varphi\rangle:=-\frac{1}{n} \int_{\Omega}(\operatorname{adj} D u \cdot u) \cdot D \varphi \quad \varphi \in \mathcal{D}(\Omega)\left({ }^{4}\right) .
$$

\footnotetext{
${ }^{3}$ We denote with $\omega_{n}$ the Lebesgue measure of the unit ball $B$ and with $\delta_{0}$ the usual Dirac distribution.

${ }^{4} \mathcal{D}(\Omega)$ is the usual space of test functions.
} 
Notice that for smooth maps we have $J u=\operatorname{det} D u$ and this can be extended by density to $u \in W_{\text {loc }}^{1, n}$. However the distribution (1.5) is well defined as far as $u$ adj $D u \in L_{\text {loc }}^{1}$, for example if $u \in W^{1, p} \cap L^{\infty}$ and $p \in(n-1, n)$. The distributional Jacobian enjoys continuity properties with respect to weak convergence and may differ from $\operatorname{det} D u$ if $p<n$. For example if $u(x)=\frac{x}{|x|}$ we have

$$
J u=\omega_{n} \delta_{0},
$$

while $\operatorname{det} D u=0$.

If $J u$ is a Radon measure and $u \in W^{1, p}$ with $p \geq n-1$ we have the following theorem (see [36] for the case $p>\frac{n^{2}}{n+1}$ and [16] for the case $\left.n-1 \leq p \leq \frac{n^{2}}{n+1}\right)$.

Theorem 1.1. Let $u \in W^{1, p}(\Omega) \cap L_{\mathrm{loc}}^{\infty}$, if Ju is a Radon measure then

$$
J u=\operatorname{det} D u \mathrm{~d} \mathcal{L}^{n}+J u^{S} .
$$

The second formulation we consider is the Lebesgue-Serrin extension of the functional (1.3) (see Sect. 2.4 for a more detailed discussion). We define for every $p \in(n-1, n)$

$$
T V^{p}(u, \Omega)=\inf \left\{\liminf _{k \rightarrow \infty} \int_{\Omega}\left|\operatorname{det} D u_{k}\right|: u_{k} \in W_{\mathrm{loc}}^{1, n}, u_{k} \rightarrow u \text { in } W^{1, p}\right\} .
$$

If $u \in W^{1, n}$ and $p>n-1$ semicontinuity results below the natural growth exponent (see [13,30,31]) ensure that:

$$
T V^{p}(u, \Omega):=\int_{\Omega}|\operatorname{det} D u| \text {. }
$$

Observe we had underlined the dependence on $p$, however in all known example we have that $T V^{p}(u, \Omega)=$ $T V^{q}(u, \Omega)$, as long as $p, q \in(n-1, n)$ and $u \in W^{1, p} \cap W^{1, q}$, it seems natural to conjecture this to be always true.

Recently it has been shown by Schmidt [43] that the relaxed total variation enjoys the following quasiconvexity property ${ }^{5}$ :

for any affine map $u_{A}$ and deformation $\varphi \in W_{0}^{1, p}$

$$
T V^{p}\left(u_{A}, \Omega\right) \leq T V^{p}\left(u_{A}+\varphi, \Omega\right)
$$

When $p \in(n-1, n), u \in L^{\infty}$ and $T V^{p}(u, \Omega)<\infty$, then $T V^{p}(u, \cdot)$ can be extended to a Radon measure on $\Omega$. Moreover in this case it is possible to show that also $J u$ is a Radon measure and that, for any open subset $A \subseteq \Omega$

$$
|J u|(A) \leq T V^{p}(u, A)
$$

where $|J u|$ is the total variation of the measure $J u$ (see $[22,23]$ and Prop. 3.2 below).

In this paper we discuss equality cases in previous inequality (Thms. 4.3 and 5.6) and we show how to obtain explicit representation formulas for the extension of general polyconvex functionals satisfying suitable growth conditions (Thms. 6.1 and 6.2).

The paper is structured as follows. In Section 2 we recall some preliminary results, in particular the notion of Brouwer degree for Sobolev maps, the measure representation theorem and a theorem of Brian White about minimal mass currents. In Section 3 we begin the comparison between $|J u|$ and $T V^{p}$ showing which are the main obstructions to equality in (1.7). In Section 4 we prove that equality holds for maps with values in the $(n-1)$-dimensional sphere. In Section 5, inspired by Müller and Spector INV condition [38], we introduce

\footnotetext{
${ }^{5}$ Recall that a map $f: \mathbb{M}^{n \times n} \rightarrow \mathbb{R}$ is said to be quasi-convex if

$$
f(\xi)|B| \leq \int_{B} f(\xi+D \varphi) \quad \forall \varphi \in C_{0}^{1}(B)
$$

i.e. every affine map is a minimizer with respect to its boundary condition.
} 
a particular class of Sobolev maps for which we are able to obtain equality in (1.7). In Section 6 we show the general relaxation result.

\section{Notations AND PRELIMINARY RESUlts}

Throughout this paper $\Omega$ is an open subset of $\mathbb{R}^{n}$ with Lipschitz boundary. Points in $\mathbb{R}^{n}$ will be denoted by $x$, their $i$ th component by $x^{i},|x|$ is the usual Euclidean norm and we will also consider the $\infty$-norm:

$$
|x|_{\infty}=\max \left\{\left|x^{i}\right|: i=1, \ldots, n\right\} .
$$

We write $B\left(x_{0}, r\right)$ for $\left\{x:\left|x-x_{0}\right|<r\right\}$ and $Q\left(x_{0}, r\right)$ for $\left\{x:\left|x-x_{0}\right|_{\infty}<r\right\}$, if it is clear from the context we will drop the dependence on $x_{0}$ and simply write $B_{r}$ and $Q_{r}$. $C$ and $M$ will be constants, possibly depending on previous ones, whose value could change from line to line. $\mathcal{L}^{n}$ and $\mathcal{H}^{k}$ will denote the $n$-dimensional Lebesgue measure and the $k$-dimensional Hausdorff measure, we will write $\mathrm{d} x$ to mean $\mathrm{d} \mathcal{L}^{n}$. Given a measure $\mu$ we denote with $\mu\left\llcorner U\right.$ the restriction of $\mu$ to $U$ and with $|\mu|$ its total variation, we write $\mu^{a}$ and $\mu^{S}$ respectively for its absolutely continuous and singular part with respect to the Lebesgue measure. If $f$ is a function $f\llcorner U$ means its restriction to $U$.

For any matrix $A \in \mathbb{M}^{n \times n}$ and vectors $v, w \in \mathbb{R}^{n}$ we will denote with:

- $A_{i}^{j}$ the entry in the $j$ th row and $i$ th column, $A^{T}$ is the transpose of $A, A \cdot v$ the usual action of a matrix on a vector and $w \cdot v$ the Euclidean scalar product between $v$ and $w$;

- $\mathcal{M}_{h}(A) \in \mathbb{R}^{\tau}$, with $\tau=\left(\begin{array}{l}n \\ h\end{array}\right)^{2}$, the vector of minors of order $h$ of $A$ (i.e. the vector formed by the determinants of all $h \times h$ submatrices of $A$ );

- $\operatorname{adj} A$ the $n \times n$ matrix that satisfies:

$$
(\operatorname{adj} A) A=(\operatorname{det} A) \operatorname{Id}
$$

i.e. the transpose of the cofactor matrix of $A$.

\subsection{Sobolev maps and precise representative}

We will denote with $W^{1, p}\left(\Omega ; \mathbb{R}^{n}\right)$ the space of Sobolev maps and we refer to [17] for its main properties and notations. In the sequel we will need to consider the restriction of a function $u$ to lower dimensional subsets, mainly to the boundary $\partial D$ of Lipschitz subsets. This can be done in two ways:

(1) considering the trace $\gamma(u) \in L^{p}(\partial D)$;

(2) considering the pointwise value of the precise representative of the class of $u$ defined by:

$$
\bar{u}(x)= \begin{cases}\lim _{r \rightarrow 0} \frac{1}{|B(x, r)|} \int_{B(x, r)} u(y) \mathrm{d} y & \text { if the limit exists } \\ 0 & \text { otherwise. }\end{cases}
$$

It turns out that $\bar{u}=u \mathcal{H}^{n-1}$-almost everywhere and $\gamma(u)$ equals $\bar{u}\llcorner\partial D$. Being this understood we will simply refer to the restriction of $u$ to $\partial D$.

Given a domain $D$ with Lipschitz boundary we say that a function is in $W^{1, p}(\partial D)$ if its restriction satisfies:

$$
\int_{\partial D}|u|^{p}+\left|D^{T} u\right|^{p} \mathrm{~d} \mathcal{H}^{n-1}<\infty
$$

where $D^{T} u(x)=D u(x)\left\llcorner T_{x}(\partial D)^{6}\right.$. The definition could equivalently be given locally by flattening the boundary. With an abuse of notation we say that $u \in W^{1, p}(\bar{D})$ if $u \in W^{1, p}(D) \cap W^{1, p}(\partial D)$.

\footnotetext{
${ }^{6} T_{x}(\partial D)$ is the tangent space defined $\mathcal{H}^{n-1}\llcorner\partial D$-almost everywhere.
} 
Proposition 2.1. If $u \in W^{1, p}(\bar{D})$ there exists a sequence of Lipschitz maps $u_{k}$ converging to $u$ in $W^{1, p}(\bar{D})$, i.e.

$$
\left\|u-u_{k}\right\|_{W^{1, p}(\bar{D})}:=\left\|u-u_{k}\right\|_{W^{1, p}(D)}+\left\|u-u_{k}\right\|_{W^{1, p}(\partial D)} \rightarrow 0 .
$$

Proof. By standard arguments we can reduce to the case where $u \in W^{1, p}\left(\overline{B^{+}}\right)$and spt $u$ is a compact subset of $B^{+}=\left\{x=\left(x^{\prime}, x^{n}\right):|x|<1, x^{n} \geq 0\right\}$. Moreover if we consider the sequence:

$$
u_{k}(x)= \begin{cases}u\left(x^{\prime}, 0\right) & \text { if } 0 \leq x^{n} \leq \frac{1}{k} \\ u\left(x^{\prime}, x^{n}-\frac{1}{k}\right) & \text { if } x^{n} \geq \frac{1}{k}\end{cases}
$$

we have that $u_{k} \rightarrow u$ in $W^{1, p}\left(\overline{B^{+}}\right)$so we may suppose $u$ to be a constant function of $x^{n}$ for small $x^{n}$, say smaller than $\delta$. Consider now a sequence of smooth maps $\left\{v_{k}\right\}$ converging to $u$ in $W^{1, p}(D)$. Then we have

$$
\int_{0}^{\delta} \int_{\mathbb{R}^{n-1}}\left|u\left(x^{\prime}, t\right)-v_{k}\left(x^{\prime}, t\right)\right|^{p}+\left|D u\left(x^{\prime}, t\right)-D v_{k}\left(x^{\prime}, t\right)\right|^{p} \mathrm{~d} x^{\prime} \mathrm{d} t \rightarrow 0 .
$$

Hence there exists an $s \in(0, \delta)$ such that, up to subsequences:

$$
\int_{\mathbb{R}^{n-1}}\left|u\left(x^{\prime}, s\right)-v_{k}\left(x^{\prime}, s\right)\right|^{p}+\left|D u\left(x^{\prime}, s\right)-D v_{k}\left(x^{\prime}, s\right)\right|^{p} \mathrm{~d} x^{\prime} \rightarrow 0 .
$$

Recalling that $u\left(x^{\prime}, 0\right)=u\left(x^{\prime}, s\right)$ it is easy to see that the sequence defined by:

$$
u_{k}(x)= \begin{cases}v_{k}\left(x^{\prime}, s\right) & \text { if } 0 \leq x^{n} \leq s \\ v_{k}\left(x^{\prime}, x^{n}\right) & \text { if } x^{n} \geq s\end{cases}
$$

satisfies $\left\|u_{k}-u\right\|_{W^{1, p}\left(\overline{B^{+}}\right)} \rightarrow 0$.

We end this section by stating the following theorem about continuity of minors (see [12], Thm. 8.20).

Theorem 2.2 (Reshetnyak). Let $u_{k} \in W^{1, p}(\Omega)$ weakly converging to $u$, if $h<p$ we have:

$$
\mathcal{M}_{h}\left(D u_{k}\right) \rightarrow \mathcal{M}_{h}(D u) \quad \text { in } L^{\frac{p}{h}}(\Omega) .
$$

\subsection{Brouwer degree}

In [38] (see also $[7,8,19,25,39,40]$ ) a definition of Brouwer degree has been given for the class of, possibly discontinuous, Sobolev maps $W^{1, p}$ with $p>n-1$ (see [11] for the case $p=n-1$ ). Here we report some of its main properties.

Recall that for a smooth map $v: \bar{\Omega} \rightarrow \mathbb{R}^{n}$ the Brouwer degree is defined for any $y \in \mathbb{R}^{n} \backslash v(\partial \Omega)$ by:

$$
\operatorname{deg}(v, \Omega, y):=\sum_{x \in v^{-1}(\{y\})} \operatorname{sign}(\operatorname{det} D v(x)) .
$$

Moreover the oriented area formula (1.2) says that for any $h \in L^{1}\left(\mathbb{R}^{n} ; \mathbb{R}\right)$ :

$$
\int_{\mathbb{R}^{n}} h(y) \operatorname{deg}(v, \Omega, y) \mathrm{d} y=\int_{\Omega} h(v(x)) \operatorname{det} D v(x) \mathrm{d} x .
$$


Recalling equation (1.4) we have for any $g \in C^{1}\left(\mathbb{R}^{n} ; \mathbb{R}^{n}\right) \cap W^{1, \infty}$ :

$$
\begin{aligned}
\int_{\mathbb{R}^{n}} \operatorname{deg}(v, \Omega, y) \operatorname{div}_{y} g(y) \mathrm{d} y & =\int_{\Omega} \operatorname{div}_{y} g(v(x)) \operatorname{det} D v(x) \mathrm{d} x \\
& =\int_{\Omega} \sum_{i} \frac{\partial g^{i}}{\partial y^{i}}(v(x)) \operatorname{det} D v(x) \mathrm{d} x=\int_{\Omega} \sum_{i, m} \frac{\partial g^{i}}{\partial y^{m}}(v(x)) \delta_{i}^{m} \operatorname{det} D v(x) \mathrm{d} x \\
& =\int_{\Omega} \sum_{i, m, k} \frac{\partial g^{i}}{\partial y^{m}}(v(x)) \frac{\partial v^{m}}{\partial x_{k}}(x)(\operatorname{adj} D v(x))_{i}^{k} \mathrm{~d} x \\
& =\int_{\Omega} \sum_{k, i} \frac{\partial}{\partial x^{k}}\left(g^{i}(v(x))\right)(\operatorname{adj} D v(x))_{i}^{k} \mathrm{~d} x \\
& =\int_{\Omega} \sum_{k, i} \frac{\partial}{\partial x^{k}}\left(g^{i}(v(x))(\operatorname{adj} D v(x))_{i}^{k}\right) \mathrm{d} x \\
& =\int_{\Omega} \operatorname{div}_{x}(\operatorname{adj} D v(x) \cdot g(v(x))) \mathrm{d} x=\int_{\partial \Omega}(\operatorname{adj} D v(x) \cdot g(v(x))) \cdot \nu_{\partial \Omega} \mathrm{d} \mathcal{H}^{n-1} .
\end{aligned}
$$

The previous equations reveal the following facts:

(1) $\operatorname{deg}(v, \Omega, y)$ depends only on $v\llcorner\partial \Omega$;

(2) $\operatorname{deg}(v, \Omega, \cdot)$ is a $B V$ function (see [17] for the definition and for the main properties of $B V$ functions), moreover:

$$
\left|D_{y}(\operatorname{deg}(v, \Omega, y))\right|\left(\mathbb{R}^{n}\right) \leq \int_{\partial \Omega}|\operatorname{adj} D u| \leq c \int_{\partial \Omega}|D u|^{n-1}
$$

and $\operatorname{spt} D_{y}(\operatorname{deg}(v, \Omega, y)) \subset v(\partial \Omega)$.

According to this we give the following definition:

Definition 2.3. Let $u \in W^{1, p}\left(\Omega, \mathbb{R}^{n}\right)$ with $p \in(n-1, n)$. For every $D \subset \subset \Omega$ with Lipschitz boundary and such that $u \in W^{1, p}(\bar{D})$, we define the degree of $u$ on $D, \operatorname{Deg}(u, D, y)$, as the only $B V\left(\mathbb{R}^{n}, \mathbb{Z}\right)$ function satisfying:

$$
\int_{\mathbb{R}^{n}} \operatorname{Deg}(u, D, y) \operatorname{div} g(y) \mathrm{d} y=\int_{\partial D}(\operatorname{adj} D u \cdot g(u(x))) \cdot \nu_{\partial D} \mathrm{~d} \mathcal{H}^{n-1}
$$

for every $g \in C^{1}\left(\mathbb{R}^{n} ; \mathbb{R}^{n}\right)$.

Using Proposition 2.1 and equation (2.3) it is easy to see that $\operatorname{Deg}(u, D, y)$ is well defined.

It is clear from the definition that $\operatorname{Deg}(u, D, y)$ depends only on the value of $u$ restricted to $\partial D$. By Sobolev embedding Theorem, we have that $u \in C^{0}(\partial D)$, and classical theorems imply that there exists a continuous function $\varphi: \bar{D} \rightarrow \mathbb{R}^{n}$ such that $\varphi\llcorner\partial D=u\llcorner\partial D$. It is possible to show (see [38]) that:

$$
\operatorname{deg}(\varphi, D, y)=\operatorname{Deg}(u, D, y) .
$$

The Brouwer degree enjoys the following continuity property:

Proposition 2.4. Let $u_{k}, u \in W^{1, p}\left(\Omega, \mathbb{R}^{n}\right)$, then for every domain $D \subset \subset \Omega$ with Lipschitz boundary such that

$$
u_{k} \rightarrow u \text { in } W^{1, p}(\partial D)
$$

we have:

$$
\operatorname{Deg}\left(u_{k}, D, \cdot\right) \rightarrow \operatorname{Deg}(u, D, \cdot) \quad \text { in } L^{1}\left(\mathbb{R}^{n}\right)
$$


Proof. Thanks to the weak convergence and the Sobolev embedding Theorem we have

$$
\sup \left\|u_{k}\right\|_{L^{\infty}(\partial D)}+\|D u\|_{L^{p}(\partial D)} \leq C .
$$

Equation (2.3) ensures that the sequence $\left\{\operatorname{Deg}\left(u_{k}, D, \cdot\right)\right\}$ is relatively compact in $L^{1}\left(\mathbb{R}^{n}\right)$. Call $d(y) \in B V\left(\mathbb{R}^{n}, \mathbb{Z}\right)$ its limit. Recalling that, thanks to our hypothesis,

$$
\left(\operatorname{adj} D u_{k}\right)^{T} \cdot \nu_{\partial D} \rightarrow(\operatorname{adj} D u)^{T} \cdot \nu_{\partial D}
$$

in $L^{\frac{p}{n-1}}(\partial D)$ and $g\left(u_{k}\right) \rightarrow g(u)$ in any $L^{q}(\partial D)$ (see Thm. 2.2) we can pass to the limit in both sides of (2.4) to show

$$
d(y)=\operatorname{Deg}(u, D, y)
$$

\subsection{White's Theorem}

In this section we report a theorem about approximation of minimal mass rectifiable current due to White [44]. We will use it only in the simplified form expressed by Proposition 2.6 which doesn't involve currents.

Recall that a $k$-current in $\mathbb{R}^{n}, T \in \mathcal{D}_{k}\left(\mathbb{R}^{n}\right)$ is defined as linear continuous functional on the space of compactly supported $k$-forms $\mathcal{D}^{k}\left(\mathbb{R}^{n}\right)$. A current $T$ is said rectifiable, $T \in \mathcal{R}_{k}\left(\mathbb{R}^{n}\right)$, if its action on a $k$-form can be expressed as:

$$
T(\omega)=\int_{M}\left\langle\omega(x), \tau_{M}(x)\right\rangle \theta(x) \mathrm{d} \mathcal{H}^{k}
$$

where $M$ is an $\mathcal{H}^{k}$-rectifiable set, $\tau_{M}(x)$ is a $k$-vector orienting $T_{x} M$ and $\theta(x)$ is an integer-valued positive function (see $[18,26]$ for a precise definition and the statements of the theorems we use in the sequel). If $T \in \mathcal{D}_{k}\left(\mathbb{R}^{n}\right)$ we define:

- the boundary $\partial T \in \mathcal{D}_{k-1}\left(\mathbb{R}^{n}\right)$ as $\partial T(\omega)=T(\mathrm{~d} \omega)$;

- the mass $\mathbb{M}(T)=\sup \{T(\omega):|\omega| \leq 1\}$;

- the push-forward by a smooth proper map $f$ as $f_{\#} T(\omega)=T\left(f^{\#} \omega\right)^{7}$.

We can now state:

Theorem 2.5 (White). Let $f: \partial B(0,1) \subset \mathbb{R}^{n} \rightarrow \mathbb{R}^{k}$ be a Lipschitz map and $3 \leq n \leq k$ then:

$$
\min \left\{\mathbb{M}(T), T \in \mathcal{R}_{n}\left(\mathbb{R}^{k}\right), \partial T=f_{\#} \llbracket \partial B \rrbracket\right\}=\inf \left\{\int_{B} J_{n} g(x) \mathrm{d} x, g: B \rightarrow \mathbb{R}^{k}, g \text { Lipschitz, gட} \partial B=f\right\}
$$

where $J_{n} g=\sqrt{\operatorname{det} D g^{T} D g}$.

Notice the restriction $n \geq 3$, which is a consequence of Hurewicz Theorem about the relation between homotopy and homology groups (see [27]). Example 3.5 shows that this restriction is sharp.

As said before we will use the theorem only in the following form:

Proposition 2.6. Let $u: \bar{B} \subset \mathbb{R}^{n} \rightarrow \mathbb{R}^{n}$ be Lipschitz, $n \geq 3$. Then for every $\sigma>0$ there exists $g: \bar{B} \rightarrow \mathbb{R}^{n}$ Lipschitz such that $g=u$ on $\partial B$ and:

$$
\int_{B}|\operatorname{det} D g(x)| \mathrm{d} x \leq \int_{\mathbb{R}^{n}}|\operatorname{deg}(u, B, y)| \mathrm{d} y+\sigma .
$$

Proof. We have only to show that:

$$
\int_{\mathbb{R}^{n}}|\operatorname{deg}(u, B, y)| \mathrm{d} y=\min \left\{\mathbb{M}(T), T \in \mathcal{R}^{n}\left(\mathbb{R}^{n}\right) \partial T=u_{\#} \llbracket \partial B \rrbracket\right\} .
$$

\footnotetext{
${ }^{7} f^{\#} \omega$ is the usual pull-back of a differential form.
} 
We will show more, namely that the current $T=u_{\#} \llbracket B \rrbracket=\mathcal{L}^{n} \operatorname{Ldeg}(u, B, y) e_{1} \wedge \ldots \wedge e_{n}$ is the only finite mass current in the class of competitors of the previous minimum.

First of all notice that $u_{\#} \llbracket \partial B \rrbracket=\partial u_{\#} \llbracket B \rrbracket$ and:

$$
\begin{aligned}
u_{\#} \llbracket B \rrbracket\left(\psi(y) \mathrm{d} y^{1} \wedge \ldots \wedge \mathrm{d} y^{n}\right) & =\llbracket B \rrbracket\left(\psi(u(x)) \mathrm{d} u^{1} \wedge \ldots \wedge \mathrm{d} u^{n}\right) \\
& =\int_{B} \psi(u(x)) \operatorname{det} D u \mathrm{~d} x=\int_{\mathbb{R}^{n}} \psi(y) \operatorname{deg}(u, B, y) \mathrm{d} y
\end{aligned}
$$

so that:

$$
\mathbb{M}(T)=\int_{\mathbb{R}^{n}}|\operatorname{deg}(u, B, y)| \mathrm{d} y .
$$

Let now $S \in \mathcal{R}^{n}\left(\mathbb{R}^{n}\right)$ be such that $\partial S=\partial T$, then by Constancy Theorem (see [18] 4.1.7) we have that there exists $c \in \mathbb{R}$ such that:

$$
S=T+c \llbracket \mathbb{R}^{n} \rrbracket
$$

and so $\mathbb{M}(S)=\infty$ unless $c=0$.

\subsection{Lebesgue-Serrin extension}

We briefly recall the definition of Lebesgue-Serrin extension of a functional below its natural growth exponent. See $[21,30,31]$ and references therein for a more detailed exposition and $[6,20]$ for the proof of the cited results.

Suppose we have a continuous function $f: \mathbb{M}^{n \times n} \rightarrow[0, \infty)$ satisfying the growth assumption:

$$
f(\xi) \leq c(1+|\xi|)^{q}
$$

and define:

$$
F(u, \Omega)=\int_{\Omega} f(D u(x))
$$

Then $F$ is finite and strongly continuous only on $W^{1, q}$ (in our case we are considering $f(\xi)=|\operatorname{det} \xi|$ and so $q=n)$. There is a standard way to extend $F$ to $W^{1, p}$ for $p<q$ by defining:

$$
\mathcal{F}^{p}(u, \Omega)=\inf \left\{\liminf _{k \rightarrow \infty} F\left(u_{k}, \Omega\right) \quad u_{k} \in W_{\mathrm{loc}}^{1, q}, u_{k} \rightarrow u \text { in } W^{1, p}\right\} .
$$

We remark that the choice of sequences in $W_{\text {loc }}^{1, q}$ prevents some problems at the boundary of $\Omega$ if this is not smooth, see [20]. Observe that if $F$ is $p$-coercive (i.e. $F(u) \geq c\|u\|_{W^{1, p}}$ ) then $\mathcal{F}^{p}$ is the largest lowersemicontinuous functional below $F$.

In general $\mathcal{F}$ cannot be (extended to) a measure in the second variable, however we have the following result:

Theorem 2.7. Let $p<q<p \frac{n}{n-1}$ and let $u \in W^{1, p}$ be such that $\mathcal{F}^{p}(u, \Omega)<\infty$. Then there exists a Radon measure $\mu_{u}^{p}$ on $\Omega$ such that:

$$
\mu_{u}^{p}(A)=\mathcal{F}^{p}(u, A)
$$

for any open set $A \subseteq \Omega$. Moreover if $f$ is a quasi-convex function we have that the Radon-Nykodim derivative of $\mu_{u}^{p}$ equals $f$, that is:

almost everywhere in $\Omega$.

$$
\frac{\mathrm{d} \mu_{u}^{p}}{\mathrm{~d} \mathcal{L}^{n}}(x)=f(D u(x))
$$

In the sequel we show, under appropriate hypotheses, how to characterize the singular part of $\mu_{u}^{p}$.

We end this section by recalling two classical theorems about continuity and semi-continuity for functionals defined on the space of Radon measures (see [2] for a proof). 
If $g: \mathbb{R} \rightarrow[0, \infty)$ is a convex function, we define the recession function as:

$$
g_{\infty}(s):=\lim _{t \rightarrow+\infty} \frac{g(s t)-g(0)}{t}
$$

which turns out to be a positively homogeneous, lower semicontinuous function. Moreover if $g(t) \leq c(1+|t|)$ we have that $g_{\infty}$ is locally bounded and hence continuous.

Theorem 2.8. Let $\mu$ be a positive Radon measure and $g: \mathbb{R} \rightarrow[0, \infty)$ be a convex function. Then the functional defined on $\mathcal{M}(\Omega ; \mathbb{R})^{8}$ by:

$$
G(\lambda)=\int_{\Omega} g\left(\frac{\mathrm{d} \lambda}{\mathrm{d} \mu}\right) \mathrm{d} \mu+\int_{\Omega} g_{\infty}\left(\frac{\mathrm{d} \lambda}{\mathrm{d}|\lambda|}\right) \mathrm{d}|\lambda|
$$

is lower semicontinuous with respect to weak-* convergence.

Theorem 2.9 (Reshetnyak). Let $h: \mathbb{R}^{n} \rightarrow[0, \infty)$ be a continuous, convex and positively homogeneous function and for any $\lambda \in \mathcal{M}\left(\Omega ; \mathbb{R}^{n}\right)$ consider:

$$
H(\lambda)=\int_{\Omega} h\left(\frac{\mathrm{d} \lambda}{\mathrm{d}|\lambda|}\right) \mathrm{d}|\lambda| .
$$

Then if $\mu_{k} \stackrel{*}{\rightarrow} \mu$ and $\left|\mu_{k}\right|(\Omega) \rightarrow|\mu|(\Omega)$ we have:

$$
H\left(\mu_{k}\right) \rightarrow H(\mu)
$$

\section{3. $T V$ VS. Ju}

First of all we show the continuity of the distributional Jacobian:

Proposition 3.1. Let $\left\{u_{k}\right\} \subset W^{1, p}(\Omega)$ such that $u_{k} \rightarrow u$, suppose $p>n-1$ and

$$
\sup _{k}\left\|u_{k}\right\|_{L^{\infty}(K)} \leq C(K)
$$

for any compact $K \subset \Omega$, then

$$
J u_{k} \stackrel{*}{\rightarrow} J u
$$

as distributions.

Proof. We have to prove that for every $\varphi \in \mathcal{D}(\Omega)$ :

$$
\sum_{j} \int_{\operatorname{spt} \varphi} u_{k}^{i}\left(\operatorname{adj} D u_{k}\right)_{i}^{j} \partial_{j} \varphi \rightarrow \sum_{j} \int_{\operatorname{spt} \varphi} u^{i}(\operatorname{adj} D u)_{i}^{j} \partial_{j}, \varphi
$$

From Theorem 2.2 we have adj $D u_{k} \rightarrow \operatorname{adj} D u$ in $L^{\frac{p}{n-1}}$, moreover the sequence is $L^{\infty}$ bounded on spt $\varphi$, hence we have $u_{k}^{i} \partial_{j} \varphi \rightarrow u^{i} \partial_{j} \varphi$ in $L^{\frac{p}{p-(n-1)}}=\left(L^{\frac{p}{n-1}}\right)^{*}$, so the thesis follows.

\footnotetext{
${ }^{8} \mathcal{M}(\Omega ; \mathbb{R})$ is the set of finite Radon measures on $\Omega$.
} 
It is clear from the proof that if $u_{k} \rightarrow u$ in $W^{1, p}$ with $p>\frac{n^{2}}{n+1}$ then $J u_{k} \stackrel{*}{\rightarrow} J u$ as distributions without any boundedness assumptions, in fact in this case Sobolev embedding theorem implies that $u_{k} \rightarrow u$ in $L^{\frac{p}{p-(n-1)}}$.

Consequence of this is the following comparison result (see $[22,23])$ :

Proposition 3.2. Suppose $p \in(n-1, n)$ and $u \in W^{1, p}\left(\Omega ; \mathbb{R}^{n}\right) \cap L_{\mathrm{loc}}^{\infty}$ satisfies $T V^{p}(u, \Omega)<\infty$, then Ju is a Radon measure and for any open subset $A \subset \Omega$ :

$$
|J u|(A) \leq T V^{p}(u, A) .
$$

Proof. Suppose $u \in L^{\infty}(\Omega)$ and let $u_{k} \in W_{\text {loc }}^{1, n}(\Omega)$ be a sequence weakly converging to $u$ and satisfying

$$
\liminf _{k \rightarrow \infty} \int_{\Omega}\left|\operatorname{det} D u_{k}\right|<\infty
$$

Applying Lemma A.1 we can find a sequence $\left\{v_{k}\right\}$, also weakly converging to $u$, such that $\left\|v_{k}\right\|_{\infty} \leq 2\|u\|_{\infty}$ and, for any open set $A \subset \Omega$,

$$
\liminf _{k \rightarrow \infty} \int_{A}\left|\operatorname{det} D v_{k}\right| \leq \liminf _{k \rightarrow \infty} \int_{A}\left|\operatorname{det} D u_{k}\right| .
$$

Then, thanks to Proposition 3.1, $\operatorname{det} D v_{k} \mathrm{~d} \mathcal{L}^{n}$ is a sequence of Radon measures with equi-bounded total variation which converges to $J u$ in the sense of distributions, hence the first part of the theorem follows. The second part is a consequence of equation (3.2) and of the semicontinuity of the total variation with respect to weak-* convergence of measures.

Let now $u$ be in $L_{\text {loc }}^{\infty}(\Omega)$, by the previous part the distribution $J u$ is a Radon measure when restricted to any open set $A \subset \subset \Omega$, moreover for any $\varphi \in \mathcal{D}(\Omega)$ we can choose an open set $U$ such that spt $\varphi \subset U \subset \subset \Omega$, then we have

$$
\langle J u, \varphi\rangle \leq\|\varphi\|_{\infty} T V^{p}(u, U) \leq\|\varphi\|_{\infty} T V^{p}(u, \Omega),
$$

and hence, thanks to the Riesz theorem, $J u$ is a Radon measure also in $\Omega$. Equation (3.1) can now be extended to any open set $A \subset \Omega$ by approximation.

Equality in (3.1) is not achieved in general. The reasons for this gap being essentially of two types: cancellation and topological obstruction. In order to show this we prove two propositions which link the Brouwer degree for Sobolev maps to the Distributional Jacobian and the Total Variation. The first one is well known (see [28]) while the second one is an immediate generalization of Theorem 1.4 of [33].

We call a map $u \in W^{1, p}\left(B(0,1) ; \mathbb{R}^{n}\right)$-homogeneous if there exists a Lipschitz map $\varphi: S^{n-1}=\partial B \rightarrow \mathbb{R}^{n}$ such that $u(x)=\varphi\left(\frac{x}{|x|}\right)$. Observe that $u \in \operatorname{Lip}_{\text {loc }}(B \backslash\{0\}) \cap W^{1, p}$ for every $p<n$.

Proposition 3.3. Let $u$ be a 0-homogeneous map, then:

$$
J u=\left(\int_{\mathbb{R}^{n}} \operatorname{Deg}(u, B, y) \mathrm{d} y\right) \delta_{0} .
$$

Proof. Let $v: B \rightarrow \mathbb{R}^{n}$ be a Lipschitz map coinciding with $\varphi$ on $\partial B$, then:

$$
\operatorname{Deg}(u, B, y)=\operatorname{deg}(v, B, y)
$$

Moreover the sequence:

$$
u_{\varepsilon}(x)= \begin{cases}\varphi\left(\frac{x}{|x|}\right) & \text { if } \varepsilon<|x|<1 \\ v\left(\frac{x}{\varepsilon}\right) & \text { if }|x|<\varepsilon\end{cases}
$$


converges to $u$ in $W^{1, p}$. Thanks to Proposition 3.1 we have:

$$
\operatorname{det} D u_{\varepsilon} \stackrel{*}{\rightarrow} J u
$$

in the sense of distributions. Let $\psi$ be a test function, then:

$$
\begin{aligned}
\int \psi(x) \operatorname{det} D u_{\varepsilon}(x) & =\frac{1}{\varepsilon^{n}} \int_{B(0, \varepsilon)} \psi(x) \operatorname{det} D v\left(\frac{x}{\varepsilon}\right) \mathrm{d} x \\
& =\int_{B(0,1)} \psi(\varepsilon x) \operatorname{det} D v(x) \mathrm{d} x \rightarrow \psi(0) \int_{B(0,1)} \operatorname{det} D v
\end{aligned}
$$

and thanks to (1.1) we have:

$$
\int_{B} \operatorname{det} D v(x) \mathrm{d} x=\int_{R^{n}} \operatorname{deg}(v, B, y) \mathrm{d} y
$$

Proposition 3.4. Let $u: B \rightarrow \mathbb{R}^{n}$ be a 0 -homogeneous map and $n \geq 3$. Then for any $p \in(n-1, n)$ :

$$
T V^{p}(u, B)=\int_{\mathbb{R}^{n}}|\operatorname{Deg}(u, B, y)| \mathrm{d} y
$$

Proof. Let $w$ be a Lipschitz map that agrees with $\varphi$ on $\partial B$. Thanks to Proposition 2.6, for any positive $\sigma$ there exists a Lipschitz map $g$ which agrees with $w$ on $\partial B$ and such that:

$$
\int_{B}|\operatorname{det} D g| \leq \int_{\mathbb{R}^{n}}|\operatorname{deg}(w, B, y)|+\sigma=\int_{\mathbb{R}^{n}}|\operatorname{Deg}(u, B, y)|+\sigma .
$$

Considering

$$
u_{\varepsilon}(x)= \begin{cases}\varphi\left(\frac{x}{|x|}\right) & \text { if } \varepsilon<|x|<1 \\ g\left(\frac{x}{\varepsilon}\right) & \text { if }|x|<\varepsilon\end{cases}
$$

we have

$$
T V^{p}(u, B) \leq \int_{\mathbb{R}^{n}}|\operatorname{Deg}(u, B, y)| \mathrm{d} y .
$$

To prove the reverse inequality we recall that for 0-homogeneous maps the approximation sequences could be chosen to satisfy $u_{k}=u$ on $\partial B$ (see [22], Lem. 22). So for any such sequence we have, thanks to the unoriented version of the area formula:

$$
\begin{aligned}
\int_{B}\left|\operatorname{det} D u_{k}\right| & =\int_{\mathbb{R}^{n}} \#\left\{x \in \Omega: u_{k}(x)=y\right\} \mathrm{d} y \\
& \geq \int_{\mathbb{R}^{n}}\left|\operatorname{deg}\left(u_{k}, B, y\right)\right| \mathrm{d} y=\int_{\mathbb{R}^{n}}|\operatorname{Deg}(u, B, y)| \mathrm{d} y
\end{aligned}
$$

It is clear now that for 0 -homogeneous maps and $n \geq 3$ a necessary and sufficient condition for equality in (3.1) is that $\operatorname{Deg}(u, B, y)$ does not change $\operatorname{sign}$ (i.e. there must not be cancellation).

The following example, considered for the first time by Malý in [29], shows that the situation for $n=2$ is more involved (see also [22-24]). Some kind of homotopic topological obstructions prevents the equality in (3.1). 
Example 3.5. Consider the following function $\varphi: S^{1} \rightarrow \mathbb{R}^{2}$ :

$$
\varphi(\vartheta):= \begin{cases}(-1+\cos 4 \vartheta, \sin 4 \vartheta) & \vartheta \in\left[0, \frac{\pi}{2}\right] \\ (1-\cos 4 \vartheta, \sin 4 \vartheta) & \vartheta \in\left[\frac{\pi}{2}, \pi\right] \\ (-1+\cos 4 \vartheta,-\sin 4 \vartheta) & \vartheta \in\left[\pi, \frac{3 \pi}{2}\right] \\ (1-\cos 4 \vartheta,-\sin 4 \vartheta) & \vartheta \in\left[\frac{3 \pi}{2}, 2 \pi\right]\end{cases}
$$

which covers the "eight" curve:

$$
\begin{aligned}
X= & \left\{\left(x^{1}, x^{2}\right) \in \mathbb{R}^{2}:\left(x^{1}-1\right)^{2}+\left(x^{2}\right)^{2}=1\right\} \\
& \cup\left\{\left(x^{1}, x^{2}\right) \in \mathbb{R}^{2}:\left(x^{1}+1\right)^{2}+\left(x^{2}\right)^{2}=1\right\}
\end{aligned}
$$

two times with opposite orientation. Define its 0 -homogeneous extension to the unit ball $u(\varrho, \vartheta):=\varphi(\vartheta)$. We have that $\operatorname{Deg}(u, B, y)=0$ and so:

$$
J u=0
$$

however:

$$
T V^{p}(u, \cdot)=2 \pi \delta_{0} .
$$

In fact, because of the non trivial homotopy type of $\varphi\left(S^{1}\right)$ in $\mathbb{R}^{2} \backslash\{(1,0),(-1,0)\}$, any smooth map approximating $u$ must cover one of the disks bounded by $X$ at least two times (see [41] for a precise discussion).

\section{SPHERE-VALUED MAPS}

In this section we show how to get equality between $J u$ and $T V^{p}$ in the case of functions whose image is contained in the $(n-1)$-dimensional sphere $S^{n-1}$. This result, conjectured in [23] and proved in [41] for 0 -homogeneous maps, is a simple consequence of Bethuel's approximation Theorem 4.2 and of the structure of Distributional Jacobian for sphere-valued maps (Thm. 4.1).

The following can be found in [28] (see also [1] for a different proof with more general hypotheses).

Theorem 4.1. Let $u \in W^{1, p}\left(\Omega ; S^{n-1}\right), p \geq n-1$ and suppose that $J u$ is a Radon measure, then exist $\left\{x_{i}\right\}_{i=1}^{m} \subset \Omega$ and $d_{i} \in \mathbb{Z}$ such that:

$$
J u=\omega_{n} \sum_{i=1}^{m} d_{i} \delta_{x_{i}}
$$

where $d_{i}=\operatorname{deg}\left(u, \partial B\left(x_{i}, r_{i}\right), S^{n-1}\right)$ with $r_{i}$ small enough.

Observe that if $p>n-1$, thanks to Sobolev immersion, $u$ L $\partial B_{r}(x)$ is continuous for almost all radii, so the degree considered in the theorem is the classical Brouwer degree of a continuous map (in the case $p=n-1$ one should refer to the theory of Brouwer degree in BMO, see [7]).

Theorem 4.2. Let $u \in W^{1, p}\left(\Omega ; S^{n-1}\right), p \geq n-1$ then there exists a sequence $\left\{u_{k}\right\} \subset C^{\infty}\left(\Omega ; S^{n-1}\right)$ strongly converging to $u$ if and only if

$$
J u=0 .
$$

This theorem has been proved for the case $p=n-1$ in [4] but the technique used there cannot be generalized. The proof for the case $p \in(n-1, n)$, instead, is an easy consequence of the techniques used in the proof of Theorem 1 of [5]. Another proof has recently been given in [42].

With these tools we are now able to prove the following:

Theorem 4.3. Let $u \in W^{1, p}\left(\Omega ; S^{n-1}\right), p>n-1$, such that $T V^{p}(u, \Omega)<\infty$, then:

$$
|J u|(\Omega)=T V^{p}(u, \Omega) .
$$


Proof. Recall that $T V^{p}(u, \Omega)<\infty$ implies that $J u$ is a Radon measure hence, thanks to Theorem 4.1, we have

$$
J u=\omega_{n} \sum_{i=1}^{m} d_{i} \delta_{x_{i}} .
$$

For simplicity we assume

$$
J u=\omega_{n} d \delta_{x_{0}}
$$

for some $x_{0} \in \Omega$ with $d=\operatorname{deg}\left(u, \partial B\left(x_{0}, r\right), S^{n-1}\right)$, the proof in the general case being equal. By translation we can suppose $x_{0}=0$.

Choose an $r<\frac{1}{2} \operatorname{dist}(0, \Omega)$, we clearly have

$$
J u(\Omega \backslash B(0, r))=0
$$

and so we can find a sequence $u_{k}$ of smooth maps with values in $S^{n-1}$ strongly converging to $u$ in $W^{1, p}(\Omega \backslash B(0, r))$. Thanks to a Fubini type argument we can find $s \in(r, 2 r)$ such that:

- $\left\|u_{k}-u\right\|_{W^{1, p}(\partial B(0, s))} \rightarrow 0$;

- $\int_{\partial B(0, s)}|D u|^{p} \leq \frac{1}{r} \int_{B(0,2 r)}|D u|^{p}$.

From Sobolev Embedding Theorem we have that $\left\|u_{k}-u\right\|_{L^{\infty}(\partial B(0, s))} \rightarrow 0$ and so, for $k$ large, we have:

$$
\operatorname{deg}\left(u_{k}, \partial B(0, s), S^{n-1}\right)=\operatorname{deg}\left(u, \partial B(0, s), S^{n-1}\right)=d
$$

Denote with $w: \partial B(0, s) \rightarrow S^{n-1}$ a smooth map such that:

$$
\begin{gathered}
\operatorname{deg}\left(w, \partial B(0, s), S^{n-1}\right)=d \\
\#\{x \in \partial B(0, s): w(x)=y\}=|d|
\end{gathered}
$$

where the last equality holds for all points in $S^{n-1}$ except two. Hopf Theorem implies that for every $k$ there exists a continuous homotopy $H_{k}: \partial B(0, s) \times[0,1] \rightarrow S^{n-1}$ such that

$$
H_{k}(\cdot, 0)=w(\cdot) \text { and } H_{k}(\cdot, 1)=u_{k}\llcorner\partial B(0, s)(\cdot)
$$

Mollifying $H_{k}$ and projecting again on $S^{n-1}$ we can suppose the homotopy to be smooth, see [41], Lemma 3 .

Let $\varrho \ll s$ and consider the map:

$$
v_{k, s, \varrho}= \begin{cases}u_{k}(x) & \text { if } x \in \Omega \backslash B(0, s) \\ u_{k}\left(s \frac{x}{|x|}\right) & \text { if } x \in B(0, s) \backslash B(0,2 \varrho) \\ H_{k}\left(s \frac{x}{|x|}, \frac{|x|-\varrho}{\varrho}\right) & \text { if } x \in B(0,2 \varrho) \backslash B(0, \varrho) \\ \frac{|x|}{\varrho} w\left(s \frac{x}{|x|}\right) & \text { if } x \in B(0, \varrho) .\end{cases}
$$


Then (recall that the area formula (1.1) implies that if $v: A \rightarrow \mathbb{R}^{n}$ is Lipschitz and $v(A) \subset S^{n-1}$ then $\operatorname{det} D v=0$ a.e. on $A)$ :

$$
\begin{aligned}
\int_{\Omega}\left|\operatorname{det} D v_{k, s, \varrho}\right| & =\int_{B(0, \varrho)}\left|\operatorname{det} D v_{k, s, \varrho}\right| \\
& =\int_{B(0, \varrho)}\left|\operatorname{det} D\left(\frac{|x|}{\varrho} w\left(s \frac{x}{|x|}\right)\right)\right| \mathrm{d} x=\int_{B(0,1)}\left|\operatorname{det} D\left(|x| w\left(s \frac{x}{|x|}\right)\right)\right| \mathrm{d} x \\
& =\int_{\mathbb{R}^{n}} \#\left\{x \in B(0,1):|x| w\left(s \frac{x}{|x|}\right)=y\right\} \mathrm{d} y \\
& =\int_{B(0,1)} \#\left\{x \in \partial B(0, s): w(x)=\frac{y}{|y|}\right\} \mathrm{d} y=\omega_{n}|d|=|J u|(\Omega),
\end{aligned}
$$

since Proposition 3.2 gives the opposite inequality to conclude the proof we just have to show that $v_{k, s, \varrho} \rightarrow u$. Actually we can show more, in fact we have strong convergence.

Observing that $\left\|v_{k, s, \varrho}\right\|_{\infty} \leq 1$ we obtain:

$$
\int_{\Omega}\left|v_{k, s, \varrho}-u\right|^{p} \leq \int_{\Omega \backslash B(0, r)}\left|u_{k}-u\right|^{p}+2^{p}|B(0, r)| \rightarrow 0
$$

for $k \rightarrow \infty$ and $r \rightarrow 0$ (recall that $s \in(r, 2 r)$ ).

Moreover:

$$
\begin{aligned}
\int_{\Omega}\left|D v_{k, s, \varrho}-D u\right|^{p} \leq \int_{\Omega \backslash B(0, r)}\left|D u_{k}-D u\right|^{p} & \\
& +C\left\{\int_{B(0, s)}|D u|^{p}+\int_{B(0, s)}\left|D\left(u_{k}\left(s \frac{x}{|x|}\right)\right)\right|^{p}+\int_{B(0,2 \varrho)}\left|D v_{k, s, \varrho}\right|^{p}\right\} .
\end{aligned}
$$

We now estimate the second and third terms inside the brackets.

The second one can be majorized as follows (see Lem. A.2):

$$
\begin{aligned}
\int_{B(0, s)} \mid D\left(\left.u_{k}\left(s \frac{x}{|x|}\right)\right|^{p}\right. & \leq C s \int_{\partial B(0, s)}\left|D u_{k}\right|^{p} \\
& \leq 2 C s \int_{\partial B(0, s)}|D u|^{p} \leq 2 C \frac{s}{r} \int_{B(0,2 r)}|D u|^{p} \leq 4 C \int_{B(0,2 r)}|D u|^{p} .
\end{aligned}
$$

Finally the last term can be estimated by:

$$
\begin{aligned}
\int_{B(0,2 \varrho)}\left|D v_{k, s, \varrho}\right|^{p} & \leq\left(\max \left\{\operatorname{Lip} H_{k}, \operatorname{Lip} w\right\}\right)^{p} \int_{B(0,2 \varrho)}\left(\frac{1}{\varrho^{p}}+s \frac{1}{|x|^{p}}\right) \\
& \leq C(n, p)\left(\max \left\{\operatorname{Lip} H_{k}, \operatorname{Lip} w\right\}\right)^{p} \varrho^{n-p}
\end{aligned}
$$

Hence, looking at (4.1), we have

$$
\int_{\Omega}\left|D v_{k, s, \varrho}-D u\right|^{p} \leq \alpha(k, r)+\beta(r)+\delta(k, \varrho)
$$


where $\beta(r) \rightarrow 0$ for $r \rightarrow 0, \alpha(k, r) \rightarrow 0$ for $k \rightarrow \infty$ (and fixed $r$ ) and $\delta(\varrho, k) \rightarrow 0$ for $\varrho \rightarrow 0$ (and fixed $k$ ). It's now clear that

$$
\left\|u-v_{k, s, \varrho}\right\|_{W^{1, p}(\Omega)} \rightarrow 0 .
$$

We remark that, thanks to the strong convergence and a standard diagonal procedure, the previous theorem gives a sequence of smooth maps such that for any open cube $Q_{R} \subset \Omega$ such that $x_{i} \notin \partial Q_{R}$ :

- $\int_{Q_{R}}\left|\operatorname{det} D v_{k}\right| \rightarrow T V^{p}\left(u, Q_{R}\right)$

- $\limsup _{k \rightarrow \infty} \int_{Q_{R}}\left|D v_{k}\right|^{p} \leq M \int_{Q_{R}}|D u|^{p}$.

Notice that this is a fine cover of $\Omega$, this fact will be used in the proof of Theorems 6.1 and 6.2.

\section{Maps With positive Degree}

According to the discussion in Section 3 we have to impose some conditions about the sign of Brouwer degree of $u$ if we want to get equality between $|J u|$ and $T V^{p}$. The following one turns out to to be sufficient.

In the sequel we will always assume $p \in(n-1, n)$.

Definition 5.1. We say that $u \in W^{1, p}(\Omega)$ weakly preserves orientation $(u \in W O P(\Omega))$ if for every $x \in \Omega$ and almost every radii $r$ :

$$
\operatorname{Deg}(u, B(x, r), y) \geq 0
$$

$W O P$ condition is inspired by the $I N V$ condition of [38] and in some sense generalizes it. In fact in [38] it is showed that if $u$ satisfies the INV condition and $\operatorname{det} D u>0$ almost everywhere, then $\operatorname{Deg}(u, B(x, r), y) \in\{0,1\}$ for almost all $r$.

If $u$ is a smooth map then $u \in \operatorname{WOP}(\Omega)$ if and only if $\operatorname{det} D u \geq 0$ in $\Omega$. For a generical Sobolev map $W O P$ condition is stronger that $\operatorname{det} D u \geq 0$ almost everywhere. Roughly speaking we are asking to preserve orientation also in the discontinuity points.

Example 5.2. Consider $u: B(0,1) \rightarrow \mathbb{R}^{2}$ :

$$
u(x)=\frac{1-|x|}{|x|}\left(-x^{1}, x^{2}\right)
$$

then $\operatorname{det} D u>0$ a.e., but $\operatorname{Deg}(u, B(0, r), y) \leq 0$, in fact a continuous map coinciding with $u$ on $\partial B(0, r)$ is the affine function:

for which $\operatorname{deg}(w, B(0, r), y) \leq 0$.

$$
w(x)=\frac{(1-r)}{r}\left(-x^{1}, x^{2}\right)
$$

Observe that the class $W O P \cap\left\{\|u\|_{\infty} \leq C\right\}$ is weakly closed. This is an immediate consequence of the continuity of degree with respect to weak convergence and of the following well-known result see [38], Lemma 2.9.

Lemma 5.3. Let $\left\{u_{k}\right\} \subset W^{1, p}(\Omega), u_{k} \rightarrow u$ then for every $x \in \Omega$ and almost every $r \in(0, \operatorname{dist}(x, \partial \Omega))$ there exists a subsequence, depending on $x$ and $r$, such that $u_{k_{j}} \rightarrow u$ in $W^{1, p}(\partial B(x, r))$.

The following proposition gives some useful properties of the distributional Jacobian of WOP maps, the proof follows the one for INV maps in [38]:

Proposition 5.4. Let $u \in W O P(\Omega) \cap W^{1, p} \cap L^{\infty}$ then:

(1) Ju is a positive Radon measure;

(2) for every $x$ and almost every $r$ :

$$
J u(B(x, r))=\int_{\mathbb{R}^{n}} \operatorname{Deg}(u, B(x, r), y) \mathrm{d} y .
$$


Proof. The first statement will follow if we are able to prove that $\left(J u \star \varrho_{\varepsilon}\right)(x) \geq 0$ where $\varrho_{\varepsilon}$ are standard mollifiers. Choose a function $f \in C^{\infty}([0,1))$ with $f^{\prime} \leq 0$ such that $\varrho_{\varepsilon}(x):=f_{\varepsilon}(|x|)$ is a family of mollifiers, where $f_{\varepsilon}(r):=\frac{1}{\varepsilon^{n}} f\left(\frac{r}{\varepsilon}\right)$

$$
\begin{aligned}
\left(J u \star \varrho_{\varepsilon}\right)(x) & =\left\langle J u, \varrho_{\varepsilon}(\cdot-x)\right\rangle \\
& =-\frac{1}{n} \int_{B(x, \varepsilon)}(\operatorname{adj} D u(y) \cdot u(y)) \cdot D \varrho_{\varepsilon}(x-y) \mathrm{d} y \\
& =-\frac{1}{n} \int_{0}^{\varepsilon} f_{\varepsilon}^{\prime}(s) \int_{\partial B(x, s)}(\operatorname{adj} D u \cdot u) \cdot \nu \mathrm{d} \mathcal{H}^{n-1} \mathrm{~d} s \\
& =-\frac{1}{n} \int_{0}^{\varepsilon} f_{\varepsilon}^{\prime}(s) \operatorname{Deg}(u, B(x, s), y) \mathrm{d} s \geq 0
\end{aligned}
$$

where in the last equality we have applied (2.4) with $g(y)=\frac{y}{n}$.

To prove the second statement choose:

$$
f_{\delta}(\varrho)= \begin{cases}1 & \varrho<r-\delta \\ \frac{r-\varrho}{\delta} & r-\delta<\varrho<r \\ 0 & \varrho>r\end{cases}
$$

then for a.e. $r$ :

$$
\begin{aligned}
J u(B(x, r)) & =\lim _{\delta \rightarrow 0}\left\langle J u, f_{\delta}(|\cdot-x|)\right\rangle \\
& =\lim _{\delta \rightarrow 0}-\frac{1}{n} \int_{B(x, r)}(\operatorname{adj} D u(y) \cdot u(y)) \cdot D_{y} f_{\delta}(|y-x|) \mathrm{d} y \\
& =\lim _{\delta \rightarrow 0} \frac{1}{n} \frac{1}{\delta} \int_{r-\delta}^{r} \int_{\partial B(x, s)}(\operatorname{adj} D u \cdot u) \cdot \nu \mathrm{d} \mathcal{H}^{n-1} \mathrm{~d} s \\
& =\frac{1}{n} \int_{\partial B(x, r)}(\operatorname{adj} D u \cdot u) \cdot \nu \mathrm{d} \mathcal{H}^{n-1}=\int_{\mathbb{R}^{n}} \operatorname{Deg}(u, B(x, r), y) \mathrm{d} y .
\end{aligned}
$$

In the sequel we will need to have a criterion to establish if equality (5.2) holds. Suppose that $u \in W^{1, p}(\Omega) \cap$ $L^{\infty}$ and $T V^{p}(u, \Omega)<\infty$. Choose a sequence $w_{n} \in C^{1}(\Omega) \cap W^{1, n}$ weakly converging to $u$ and such that

$$
\limsup _{n \rightarrow \infty} \int_{\Omega}\left|\operatorname{det} D w_{n}\right| \leq C
$$

Notice that you can also suppose $\sup \left\|w_{n}\right\|_{\infty} \leq C$ without loss of generality (see the proof of Thm. 5 in [23]). Then (up to subsequences) there exists a positive Radon measure $\sigma$ such that

$$
\operatorname{det} D w_{n} \mathrm{~d} \mathcal{L}^{n} \stackrel{*}{\rightarrow} J u \text { and }\left|\operatorname{det} D w_{n}\right| \mathrm{d} \mathcal{L}^{n} \stackrel{*}{\rightarrow} \sigma .
$$

Now for any Lipschitz $D \subset \Omega$ such that

$$
\sigma(\partial D)=0 \text { and } w_{n} \rightarrow u \text { in } W^{1, p}(\partial D)
$$


we have

$$
\begin{aligned}
J u(D) & =\lim _{n} \int_{D} \operatorname{det} D w_{n} \\
& =\lim _{n} \int_{\mathbb{R}^{n}} \operatorname{deg}\left(w_{n}, D, y\right) \mathrm{d} y=\int_{\mathbb{R}^{n}} \operatorname{Deg}(u, D, y) \mathrm{d} y
\end{aligned}
$$

thanks to Proposition 2.4.

We will need the following lemma due to Bethuel [5].

Lemma 5.5. Let $Q=[0, R]^{n}, u \in W^{1, p}(\bar{Q})$. Then there exists a constant $M$ such that for almost every $r>0$ small enough we can find a partition of $Q$ in $n_{r}=O\left(\frac{1}{r^{n}}\right)$ cubes $Q_{r}^{(i)}$ with edge $r$ and $n_{r}^{\prime}=O\left(\frac{1}{r^{n-1}}\right)$ parallelepipeds $P_{r}^{(i)}$, with edges $l_{j} \in\left[\frac{r}{4}, \frac{7 r}{4}\right], j=1, \ldots, n$ such that:

$$
\sum_{i=1}^{n_{r}} \int_{\partial Q_{r}^{(i)}}|D u|^{p} \mathrm{~d} \mathcal{H}^{n-1}+\sum_{i=1}^{n_{r}^{\prime}} \int_{\partial P_{r}^{(i)}}|D u|^{p} \mathrm{~d} \mathcal{H}^{n-1} \leq \frac{M}{r} \int_{Q}|D u|^{p} \mathrm{~d} x .
$$

Moreover we can suppose $u \mathbf{L} \partial Q_{r}^{i} \in W^{1, p}\left(\partial Q_{r}^{i}\right)$ (respectively $u\left\llcorner\partial P_{r}^{i} \in W^{1, p}\left(\partial P_{r}^{i}\right)\right)$.

Proof. Let $r \in(0, R), t \in\left(\frac{r}{4}, \frac{3 r}{4}\right)$ and $i \in\{1, \ldots, n\}$, define

$$
H_{r, i, t}=\left\{x \in Q: x^{i}=t+j r, \quad j=0, \ldots,\left\lfloor\frac{R-r}{r}\right\rfloor\right\} .
$$

Fubini's Theorem implies that for every $i$ :

$$
\int_{\frac{r}{4}}^{\frac{3 r}{4}}\left(\int_{H_{r, i, t}}|D u|^{p} \mathrm{~d} \mathcal{H}^{n-1}\right) \mathrm{d} t \leq \int_{Q}|D u|^{p} \mathrm{~d} x
$$

and so there exists $t(i) \in\left(\frac{r}{4}, \frac{3 r}{4}\right)$ such that:

$$
\int_{H_{r, i, t(i)}}|D u|^{p} \mathrm{~d} \mathcal{H}^{n-1} \leq \frac{2}{r} \int_{Q}|D u|^{p} \mathrm{~d} x
$$

Repeating this procedure for every direction $i=1, \ldots, n$ we obtain the desired partition of $Q$, in fact:

$$
\begin{aligned}
& \sum_{i=1}^{n_{r}} \int_{\partial Q_{r}^{(i)}}|D u|^{p} \mathrm{~d} \mathcal{H}^{n-1}+\sum_{i=1}^{n_{r}^{\prime}} \int_{\partial P_{r}^{(i)}}|D u|^{p} \mathrm{~d} \mathcal{H}^{n-1} \\
& \leq \frac{2 n}{r} \int_{Q}|D u|^{p} \mathrm{~d} x+\int_{\partial Q}|D u|^{p} \mathrm{~d} \mathcal{H}^{n-1} \leq \frac{2 n+1}{r} \int_{Q}|D u|^{p} \mathrm{~d} x
\end{aligned}
$$

if $r$ is small enough.

In the sequel we are going to denote both the cubes and the parallelepipeds with the improper name of cubes. Observe that for each parallelepiped there exists an affine bijection $L: P_{r}^{i} \rightarrow[0, r]^{n}$ such that the Lipschitz constant of $L$ (and of $L^{-1}$ ) is bounded by an universal constant not depending on $r$.

The following is the main result of this section. 
Theorem 5.6. Let $u \in W^{1, p}(\Omega) \cap L_{\mathrm{loc}}^{\infty}, p \in(n-1, n)$ and suppose $n \geq 3$ and $u \in W O P$ and $T V^{p}(u, \Omega)<\infty$. Then:

$$
|J u|(\Omega)=T V^{p}(u, \Omega) .
$$

Moreover for any cube $Q_{R} \subset \subset \Omega$ such that $u \in W^{1, p}\left(\bar{Q}_{R}\right)$, there exists a sequence of Lipschitz maps $v_{k} \rightarrow u$ such that:

$$
\int_{Q_{R}}\left|\operatorname{det} D v_{k}\right| \rightarrow T V^{p}\left(u, Q_{R}\right)=J u\left(Q_{R}\right)
$$

and

$$
\limsup _{k \rightarrow \infty} \int_{Q_{R}}\left|D v_{k}\right|^{p} \leq M \int_{Q_{R}}|D u|^{p}
$$

where $M$ is a universal constant not depending on $R$.

Proof. Theorem 2.7 implies that there exist a Radon measure $\mu_{u}$ such that

$$
T V^{p}(u, A)=\mu_{u}^{p}(A)
$$

for any open subset of $A \subset \Omega$. So by Besicovitch covering theorem it is sufficient to show that

$$
T V^{p}\left(u, Q_{R}\right)=\mu_{u}^{p}\left(Q_{R}\right)=J u\left(Q_{R}\right)
$$

holds for any cube $Q_{R} \subset \subset \Omega$ such that $u \in W^{1, p}\left(\bar{Q}_{R}\right)$.

Since $R$ is fixed we will denote $Q_{R}$ simply by $Q$. Recalling Proposition 3.2 we have just to show that

$$
T V^{p}(u, Q) \leq J u(Q) .
$$

By Proposition 2.1 we can find a sequence of Lipschitz maps $\left\{u_{h}\right\}$ converging to $u$ in $W^{1, p}(\bar{Q})$ and satisfying:

(1) $\left\|u_{h}\right\|_{L^{\infty}(Q)} \leq\|u\|_{L^{\infty}(Q)}$;

(2) $\int_{Q}\left|D u_{h}\right|^{p} \leq 2 \int_{Q}|D u|^{p}$

(3) $\sum_{h}\left\|u_{h}-u\right\|_{W^{1, p}}<\infty$.

Using Lemma 5.5 we can find a sequence of radii $r_{k} \rightarrow 0$ and a partition of $Q_{R}$ in $n_{k}=\left(C \frac{R}{r_{k}}\right)^{n}$ small cubes $Q_{r_{k}}^{i}$ with edges at most $\frac{7 r_{k}}{2}$ for which:

(1) $J u\left(Q_{r_{k}}^{i}\right)=\int \operatorname{Deg}\left(u, Q_{r_{k}}^{i}, y\right) \mathrm{d} y$;

(2) $J u\left(\bigcup_{i} \partial Q_{r_{k}}^{i}\right)=0$;

(3) $\sum \int_{\partial Q_{r_{k}}^{i}}|D u|^{p} \leq \frac{M}{r_{k}} \int_{Q}|D u|^{p}$;

(4) $u_{h} \rightarrow u$ in $W^{1, p}\left(\bigcup \partial Q_{r_{k}}^{i}\right)$. 
The only point that it is not immediate is the first one but it follows from the remark after Proposition 5.4. In fact it is easy to see that the sequence of radii $r_{k}$ could be chosen such that

$$
\sigma\left(\partial Q_{r_{k}}^{i}\right)=0 \text { and } w_{n} \rightarrow u \text { in } W^{1, p}\left(\partial Q_{r_{k}}^{i}\right)
$$

where $w_{n}$ is the same sequence appearing in the remark.

Thanks to Proposition 2.4 for every $k$ we can find an $h(k)$ for which:

(1) $\left\|u_{h(k)}-u\right\|_{L^{\infty}\left(\cup \partial Q_{r_{k}}^{i}\right)} \leq \frac{1}{k}$;

(2) $\int\left|\operatorname{Deg}\left(u, Q_{r_{k}}^{i}, y\right)-\operatorname{deg}\left(u_{h(k)}, Q_{r_{k}}^{i}, y\right)\right| \leq \frac{1}{k n_{k}}$ for $i=1, \ldots, n_{k}$.

We now construct the sequence of Lipschitz maps that satisfies the claim of the theorem on every small cube of the partition. For simplicity we make the construction only on the inner cubes (which are cubes with edge $2 r_{k}$ and hence of the form $Q\left(x_{i}, r_{k}\right)$ ). For the ones at the boundary, which are actually parallelepipeds, we have to do same work considering in place of $u\left\llcorner Q_{r_{k}}^{i}\right.$ the map $v=u\left\llcorner Q_{r_{k}}^{i} \circ L\right.$, where $L$ is the affine function mapping $Q_{r_{k}}^{i}$ to $\left[0, r_{k}\right]^{n}$, it is easy to see that everything still works.

For $k \in \mathbb{N}$ and $i=1, \ldots, n_{k}$ we apply Proposition 2.6 to the map $u_{h(k)}\left\llcorner Q_{r_{k}}^{i}\right.$ and obtain, for every positive $\sigma$, a Lipschitz map $g_{k}^{i}$ such that $g_{k}^{i}=u_{h(k)}$ on $\partial Q_{r_{k}}^{i}$ and

$$
\int_{Q_{r_{k}}^{i}}\left|\operatorname{det} D g_{k}^{i}\right| \leq \int\left|\operatorname{deg}\left(u_{h(k)}, Q_{r_{k}}^{i}, y\right)\right| \mathrm{d} y+\frac{\sigma}{n_{k}}
$$

Define now on $Q_{r_{k}}^{i}$ :

$$
v_{k}(x)= \begin{cases}u_{h(k)}\left(r_{k} \frac{x-x_{i}}{\left|x-x_{i}\right|_{\infty}}+x_{i}\right) & \text { if } \varrho_{k} \leq\left|x-x_{i}\right|_{\infty} \leq r_{k} \\ g_{k}^{i}\left(\frac{r_{k}}{\varrho_{k}} x\right) & \text { if }\left|x-x_{i}\right|_{\infty} \leq \varrho_{k}\end{cases}
$$

where $x_{i}$ is the center of the cube, and $\varrho_{k}$ is a sequence to be chosen later.

Observe now that:

$$
\begin{aligned}
\int_{Q}\left|\operatorname{det} D v_{k}\right| & =\sum_{i=1}^{n_{k}} \int_{Q_{r_{k}}^{i}}\left|\operatorname{det} D v_{k}\right| \\
& =\sum_{i=1}^{n_{k}}\left(\frac{r_{k}}{\varrho_{k}}\right)^{n} \int_{Q_{\varrho_{k}}^{i}}\left|\operatorname{det} D g_{k}^{i}\left(\frac{r_{k}}{\varrho_{k}} x\right)\right| \mathrm{d} x=\sum_{i=1}^{n_{k}} \int_{Q_{r_{k}}^{i}}\left|\operatorname{det} D g_{k}^{i}(x)\right| \mathrm{d} x \\
& \leq \sum_{i=1}^{n_{k}} \int_{\mathbb{R}^{n}}\left|\operatorname{deg}\left(u_{h(k)}, Q_{r_{k}}^{i}, y\right)\right| \mathrm{d} y+\sigma \leq \sum_{i=1}^{n_{k}} \int_{\mathbb{R}^{n}} \operatorname{Deg}\left(u, Q_{r_{k}}^{i}, y\right) \mathrm{d} y+\frac{1}{k}+\sigma \\
& =\sum_{i=1}^{n_{k}} J u\left(Q_{r_{k}}^{i}\right)+\frac{1}{k}+\sigma=J u(Q)+\frac{1}{k}+\sigma
\end{aligned}
$$

so the first claim will follow if we are able to show that $v_{k} \rightarrow u$. 
First of all we notice that we can suppose $\left\|g_{k}^{i}\right\|_{\infty} \leq 2\|u\|_{\infty}$, in fact thanks to Lemma A.1 we can truncate the sequence in $\left[-2\|u\|_{\infty}, 2\|u\|_{\infty}\right]^{n}$ without increasing the value of:

$$
\begin{aligned}
& \int_{Q_{r_{k}}^{i}}\left|\operatorname{det} D g_{k}^{i}\right| \\
& \int_{Q}\left|v_{k}-u\right|^{p}=\sum_{i=1}^{n_{k}} \int_{Q_{r_{k}}^{i}}\left|v_{k}-u\right|^{p} \\
& \leq \sum_{i=1}^{n_{k}} C\left\{\int_{Q_{r_{k}}^{i}}\left|u-u_{h(k)}\right|^{p}+\varrho_{k}^{n}\left\|g_{k}^{i}\right\|_{\infty}^{p}\right. \\
& \left.+\int_{Q_{r_{k}}^{i}}\left|u_{h(k)}(x)-u_{h(k)}\left(r_{k} \frac{x-x_{i}}{\left|x-x_{i}\right|_{\infty}}+x_{i}\right)\right|^{p} \mathrm{~d} x\right\} \\
& \leq C\left\{\int_{Q}\left|u-u_{h(k)}\right|^{p}+\left(\frac{\varrho_{k}}{r_{k}}\right)^{n}\|u\|_{\infty}^{p}\right. \\
& \left.+\sum_{i=1}^{n_{k}} \int_{Q_{r_{k}}^{i}}\left|u_{h(k)}(x)-u_{h(k)}\left(r_{k} \frac{x-x_{i}}{\left|x-x_{i}\right|_{\infty}}+x_{i}\right)\right|^{p} \mathrm{~d} x\right\} \\
& \leq C\left\{\int_{Q}\left|u-u_{h(k)}\right|^{p}+\left(\frac{\varrho_{k}}{r_{k}}\right)^{n}\|u\|_{\infty}^{p}+\sum_{i=1}^{n_{k}} r_{k}^{p} \int_{Q_{r_{k}}^{i}}\left|D u_{h(k)}\right|^{p} \mathrm{~d} x\right\} \\
& \leq C\left\{\int_{Q}\left|u-u_{h(k)}\right|^{p}+\left(\frac{\varrho_{k}}{r_{k}}\right)^{n}\|u\|_{\infty}^{p}+r_{k}^{p} \int_{Q}|D u|^{p}\right\}
\end{aligned}
$$

where we have used the fact that $n_{k}=O\left(\frac{1}{r_{k}^{n}}\right)$ in the second inequality and Lemma A.3 in the third one.

Consider now the gradient of $v_{k}$ :

$$
\begin{aligned}
\int_{Q}\left|D v_{k}\right|^{p} & =\sum_{i=1}^{n_{k}} \int_{Q_{r_{k}}^{i}}\left|D v_{k}\right|^{p} \\
& \leq \sum_{i=1}^{n_{k}} \int_{Q_{r_{k}}^{i}}\left|D_{x}\left(u_{h(k)}\left(r_{k} \frac{x-x_{i}}{\left|x-x_{i}\right|_{\infty}}+x_{i}\right)\right)\right|^{p}+2^{n} n_{k}\left(\frac{r_{k}}{\varrho_{k}}\right)^{p}\left(\varrho_{k}\right)^{n} \max _{i \in\left\{1, \ldots, n_{k}\right\}} \operatorname{Lip}\left(g_{k}^{i}\right)^{p} \\
& \leq \frac{1}{n-p} \sum_{i=1}^{n_{k}} r_{k} \int_{\partial Q_{r_{k}}^{i}}\left|D u_{h(k)}\right|^{p}+C(n, R)\left(\frac{\varrho_{k}}{r_{k}}\right)^{n-p} \max _{i \in\left\{1, \ldots, n_{k}\right\}} \operatorname{Lip}\left(g_{k}^{i}\right)^{p} \\
& \leq \frac{2}{n-p} \sum_{i=1}^{n_{k}} r_{k} \int_{\partial Q_{r_{k}}^{i}}|D u(x)|^{p}+C(n, R)\left(\frac{\varrho_{k}}{r_{k}}\right)^{n-p} \max _{i \in\left\{1, \ldots, n_{k}\right\}} \operatorname{Lip}\left(g_{k}^{i}\right)^{p} \\
& \leq M \int_{Q}|D u|^{p}+C(n, R)\left(\frac{\varrho_{k}}{r_{k}}\right)^{n-p} \max _{i \in\left\{1, \ldots, n_{k}\right\}} \operatorname{Lip}\left(g_{k}^{i}\right)^{p} .
\end{aligned}
$$


If we choose $\varrho_{k}$ to satisfy:
(1) $\left(\frac{\varrho_{k}}{r_{k}}\right)^{n-p} \max _{i \in\left\{1, \ldots, n_{k}\right\}} \operatorname{Lip}\left(g_{k}^{i}\right)^{p} \rightarrow 0$;
(2) $\left(\frac{\varrho_{k}}{r_{k}}\right)^{n} \rightarrow 0$

we have that $v_{k} \rightarrow u$ and so the first claim is proven. Moreover with the previous choice we have:

$$
\limsup _{k \rightarrow \infty} \int_{Q}\left|D v_{k}\right|^{p} \leq M \int_{Q}|D u|^{p}
$$

and so also the equi-boundedness hypothesis is satisfied.

\section{General relaxation Results}

In this section we give some closed formulas for the relaxed functional $\mathcal{F}^{p}(u)$ when the hypothesis of Theorems 5.6 and 4.3 are satisfied. This extends some results for radial maps proved in [31], see also [22,23].

Theorem 6.1. Let $g: \mathbb{R} \rightarrow[0, \infty)$ be a convex function such that:

$$
a|t| \leq g(t) \leq b(1+|t|)
$$

Consider:

and the relaxed functional:

$$
G(u)=\int_{\Omega} g(\operatorname{det} D u)
$$

$$
\mathcal{G}(u, \Omega)=\inf \left\{\liminf _{k \rightarrow \infty} G\left(u_{k}\right), u_{k} \in W_{\mathrm{loc}}^{1, n}, u_{k} \rightarrow u \in W^{1, p}\right\} .
$$

If $u \in W^{1, p}\left(\Omega ; \mathbb{R}^{n}\right) \cap L_{\mathrm{loc}}^{\infty}$ with $p>n-1$ satisfies $\mathcal{G}(u, \Omega)<\infty$ and

$$
u \in W^{1, p}\left(\Omega ; S^{n-1}\right)
$$

or

$$
u \in W O P(\Omega) \text { and } n \geq 3
$$

then we have:

$$
\mathcal{G}(u, \Omega)=\int_{\Omega} g(\operatorname{det} D u)+\int_{\Omega} g_{\infty}\left(\frac{\mathrm{d}(J u)^{S}}{\mathrm{~d}\left|(J u)^{S}\right|}\right) \mathrm{d}\left|(J u)^{S}\right| .
$$

Proof. Thanks to Theorem 2.7 there exists a Radon measure $\mu_{u}$ such that for every open subset $A \subseteq \Omega$

$$
\mathcal{G}(u, A)=\mu_{u}(A) \text {. }
$$

Using again Theorem 2.7 we only need to show that

$$
\mu_{u}^{S}=g_{\infty}\left(\frac{\mathrm{d}(J u)^{S}}{\mathrm{~d}\left|(J u)^{S}\right|}\right) \mathrm{d}\left|(J u)^{S}\right|
$$


in fact, in this case

$$
\begin{aligned}
\mathcal{G}(u, \Omega) & =\mu_{u}(\Omega)=\mu_{u}^{a}(\Omega)+\mu_{u}^{S}(\Omega) \\
& =\int_{\Omega} g(\operatorname{det} D u)+\int_{\Omega} g_{\infty}\left(\frac{\mathrm{d}(J u)^{S}}{\mathrm{~d}\left|(J u)^{S}\right|}\right) \mathrm{d}\left|(J u)^{S}\right|
\end{aligned}
$$

From now on suppose $u \in L^{\infty}$.

Step 1. Lower bound.

Equation (6.1) implies that $T V^{p}(u, \Omega)<\infty$ and so $J u$ is a Radon measure. Thanks to Lemma A.1, for any sequence $u_{k} \in W_{\text {loc }}^{1, n}$ weakly converging to $u$ we can find a sequence $v_{k} \in W_{\text {loc }}^{1, n}$ still converging to $u$ such that

$$
\liminf _{k \rightarrow \infty} \int_{A} g\left(\operatorname{det} D v_{k}\right) \leq \liminf _{k \rightarrow \infty} \int_{A} g\left(\operatorname{det} D u_{k}\right)
$$

for any open set $A \subset \Omega$ and $\left\|v_{k}\right\|_{\infty} \leq 2\|u\|_{\infty}$. According to Proposition 3.1 we have that

$$
\operatorname{det} D v_{k} \mathrm{~d} \mathcal{L}^{n} \stackrel{*}{\rightarrow} J u
$$

as Radon measures. Consider now the functional

$$
H(u, A)=\int_{A} g(\operatorname{det} D u(x)) \mathrm{d} x+\int_{A} g_{\infty}\left(\frac{\mathrm{d}(J u)^{S}}{\mathrm{~d}\left|(J u)^{S}\right|}\right) \mathrm{d}\left|(J u)^{S}\right| .
$$

Since, thanks to Theorem 1.1, $(J u)^{a}=\operatorname{det} D u \mathrm{~d} \mathcal{L}^{n}$ we can apply Theorem 2.8 to obtain (recall that if $v$ is in $W_{\text {loc }}^{1, n}$ then $\left.(J v)^{S}=0\right)$

$$
\begin{aligned}
\liminf _{k \rightarrow \infty} G\left(u_{k}, A\right) & =\liminf _{k \rightarrow \infty} \int_{A} g\left(\operatorname{det} D u_{k}\right) \\
& \geq \liminf _{k \rightarrow \infty} \int_{A} g\left(\operatorname{det} D v_{k}\right) \\
& =\liminf _{k \rightarrow \infty} H\left(v_{k}, A\right) \geq H(u, A) \\
& =\int_{A} g(\operatorname{det} D u(x)) \mathrm{d} x+\int_{A} g_{\infty}\left(\frac{\mathrm{d}(J u)^{S}}{\mathrm{~d}\left|(J u)^{S}\right|}\right) \mathrm{d}\left|(J u)^{S}\right|
\end{aligned}
$$

and so, taking the infimum on all sequences, the lower bound holds.

Step 2. Upper bound.

Thanks to (a variant of) Besicovitch covering Theorem, for every $\varepsilon>0$ we can find a finite family of disjoint cubes $\left\{Q_{R_{i}}^{i}\right\}$ contained in $A$ such that $u \in W^{1, p}\left(\bar{Q}_{R_{i}}\right)$ and:

$$
\mu_{u}(A) \leq \sum_{i} \mu_{u}\left(Q_{R_{i}}^{i}\right)+\varepsilon
$$

Consider now for every cube $Q_{R_{i}}^{i}$ the sequence of maps $\left\{u_{k}\right\} \in W^{1, n}\left(Q_{R_{i}}^{i}\right)$ provided by Theorems 5.6 and 4.3. This sequence satisfies:

$$
\operatorname{det} D u_{k} \mathrm{~d} \mathcal{L}^{n} \stackrel{*}{\rightarrow} J u
$$


and

$$
\int_{Q_{R_{i}}^{i}}\left|\operatorname{det} D u_{k}\right| \rightarrow|J u|\left(Q_{R_{i}}^{i}\right) .
$$

So we have:

$$
\begin{aligned}
\mu_{u}\left(Q_{R_{i}}^{i}\right) & \leq \liminf _{k \rightarrow \infty} \int_{Q_{R_{i}}^{i}} g\left(\operatorname{det} D u_{k}\right) \\
& \leq \limsup _{k \rightarrow \infty}\left\{g(0)\left|Q_{R_{i}}^{i}\right|+\int_{Q_{R_{i}}^{i}} g_{\infty}\left(\operatorname{det} D u_{k}(x)\right) \mathrm{d} x\right\} \\
& =g(0)\left|Q_{R_{i}}^{i}\right|+\int_{Q_{R_{i}}^{i}} g_{\infty}\left(\frac{\mathrm{d}(J u)}{\mathrm{d}|(J u)|}\right) \mathrm{d}|(J u)| \\
& \leq g(0)\left|Q_{R_{i}}^{i}\right|+\int_{Q_{R_{i}}^{i}} g_{\infty}\left(\frac{\mathrm{d}(\operatorname{det} D u)}{\mathrm{d}|\operatorname{det} D u|}\right) \mathrm{d}|\operatorname{det} D u|+\int_{Q_{R_{i}}^{i}} g_{\infty}\left(\frac{\mathrm{d}(J u)^{S}}{\mathrm{~d}\left|(J u)^{S}\right|}\right) \mathrm{d}\left|(J u)^{S}\right| \\
& =g(0)\left|Q_{R_{i}}^{i}\right|+\int_{Q_{R_{i}}^{i}} g_{\infty}(\operatorname{det} D u(x)) \mathrm{d} x+\int_{Q_{R_{i}}^{i}} g_{\infty}\left(\frac{\mathrm{d}(J u)^{S}}{\mathrm{~d}\left|(J u)^{S}\right|}\right) \mathrm{d}\left|(J u)^{S}\right|
\end{aligned}
$$

where we have used Reshetnyak continuity Theorem 2.9 , the sub-additivity of $g_{\infty}$, the trivial inequality

$$
g(t) \leq g(0)+g_{\infty}(t)
$$

and the fact that if $\nu$ and $\lambda$ are mutually singular measures then

$$
\frac{\mathrm{d}(\nu+\lambda)}{\mathrm{d}|\nu+\lambda|}=\frac{\mathrm{d} \nu}{\mathrm{d}|\nu|}
$$

$|\nu|-$ almost everywhere.

Then we have:

$$
\begin{aligned}
\mu_{u}(A) & \leq \sum_{i} \mu_{u}\left(Q_{R_{i}}^{i}\right)+\varepsilon \\
& \leq \sum_{i}\left\{g(0)\left|Q_{R_{i}}^{i}\right|+\int_{Q_{R_{i}}^{i}} g_{\infty}(\operatorname{det} D u(x)) \mathrm{d} x+\int_{Q_{R_{i}}^{i}} g_{\infty}\left(\frac{\mathrm{d}(J u)^{S}}{\mathrm{~d}\left|(J u)^{S}\right|}\right) \mathrm{d}\left|(J u)^{S}\right|\right\}+\varepsilon \\
& \leq g(0)|A|+\int_{A} g_{\infty}(\operatorname{det} D u(x)) \mathrm{d} x+\int_{A} g_{\infty}\left(\frac{\mathrm{d}(J u)^{S}}{\mathrm{~d}\left|(J u)^{S}\right|}\right) \mathrm{d}\left|(J u)^{S}\right|+\varepsilon .
\end{aligned}
$$

Letting $\varepsilon \rightarrow 0$ and taking singular parts we get the desired upper bound.

Consider now $u \in L_{\mathrm{loc}}^{\infty}$, since $T V^{p}(u, \Omega)<\infty$ Proposition 3.2 implies that $J u$ is a Radon measure in $\Omega$. Thanks to the previous part of the theorem we know that equation (6.2) holds for any open set $A \subset \subset \Omega$ and, since both members are Radon measures, by approximation it holds for $\Omega$.

We can also prove the following theorem:

Theorem 6.2. Let $f: \mathbb{M}^{n \times n} \rightarrow \mathbb{R}$ be a positive quasi-convex function satisfying the growth estimate:

$$
g(\operatorname{det} \xi) \leq f(\xi) \leq c\left(1+|\xi|^{p}\right)+g(\operatorname{det} \xi)
$$


where $g: \mathbb{R} \rightarrow[0, \infty)$ is a convex function satisfying the assumptions of Theorem 6.1 . If $u \in W^{1, p}\left(\Omega ; \mathbb{R}^{n}\right) \cap L_{\mathrm{loc}}^{\infty}$ with $p>\frac{n^{2}}{n+1}$ satisfies $\mathcal{F}(u, \Omega)<\infty$ and

or

$$
u \in W^{1, p}\left(\Omega ; S^{n-1}\right)
$$

$$
u \in W O P(\Omega) \text { and } n \geq 3
$$

then:

$$
\mathcal{F}(u, \Omega)=\int_{\Omega} f(D u)+\int_{\Omega} g_{\infty}\left(\frac{\mathrm{d}(J u)^{S}}{\mathrm{~d}\left|(J u)^{S}\right|}\right) \mathrm{d}\left|(J u)^{S}\right| .
$$

Proof. Since $p>\frac{n^{2}}{n+1}$ the remark after Proposition 3.1 implies that any admissible sequence $u_{k} \rightarrow u$ with bounded energy satisfies

$$
J u_{k} \stackrel{*}{\rightarrow} J u
$$

as Radon measures. Consider now the measure $\mu_{u}$, given by Theorem 2.7 which represents the relaxed functional $\mathcal{F}$, following the same lines of the proof of Theorem 6.1 and recalling that sequences given by Theorems 5.6 and 4.3 satisfy

$$
\limsup _{k \rightarrow \infty} \int_{Q}\left|D u_{k}\right|^{p} \leq M \int_{Q}|D u|^{p}
$$

we obtain, for any open subset $A \subset \subset \Omega$, the bounds

$$
\int_{A} g(\operatorname{det} D u(x)) \mathrm{d} x+\int_{A} g_{\infty}\left(\frac{\mathrm{d}(J u)^{S}}{\mathrm{~d}\left|(J u)^{S}\right|}\right) \mathrm{d}\left|(J u)^{S}\right| \leq \mu_{u}(A)
$$

and

$$
\begin{aligned}
\mu_{u}(A) \leq & c M \int_{A}\left(1+|D u|^{p}\right)+g(0)|A|+\int_{A} g_{\infty}(\operatorname{det} D u(x)) \mathrm{d} x \\
& +\int_{A} g_{\infty}\left(\frac{\mathrm{d}(J u)^{S}}{\mathrm{~d}\left|(J u)^{S}\right|}\right) \mathrm{d}\left|(J u)^{S}\right| .
\end{aligned}
$$

Taking the singular parts we obtain:

$$
\mu_{u}^{s}=g_{\infty}\left(\frac{\mathrm{d}(J u)^{S}}{\mathrm{~d} \mid(J u)^{S \mid}}\right) \mathrm{d}\left|(J u)^{S}\right| .
$$

Since Theorem 2.7 gives

$$
\mu_{u}^{a}=f(D u) \mathrm{d} \mathcal{L}^{n}
$$

we obtain the claim exactly as in Theorem 6.1 .

A careful inspection of the proof of Theorem 5.6 shows that in the previous theorem the assumption $u \in L_{\text {loc }}^{\infty}$ can be dropped.

We remark that this theorem applies to functionals related to non-linear elasticity whose model case is:

$$
F(u)=\int_{\Omega} g(\operatorname{det} D u)+|D u|^{p}
$$

and to Geometric Measure Theory, as the area of the graph of $u$ :

$$
\mathcal{A}(u)=\int_{\Omega}|\mathcal{M}(D u)|
$$

where $\mathcal{M}(D u)$ is the $n$-vector tangent to the graph of $u$. 


\section{A. Appendix}

We collect here some useful results.

Lemma A.1. Let $g: \mathbb{R} \rightarrow[0, \infty)$. Let $u \in W^{1, p}(\Omega) \cap L^{\infty}$ and $u_{k} \in W_{\text {loc }}^{1, n}(\Omega)$ a sequence weakly converging to $u$ in $W^{1, p}$ and satisfying

$$
\liminf _{k \rightarrow \infty} \int_{\Omega} g\left(\operatorname{det} D u_{k}\right)<\infty,
$$

then there exists a sequence $v_{k} \in W_{\text {loc }}^{1, n}(\Omega)$ weakly converging to $u$ such that $\left\|v_{k}\right\|_{\infty} \leq 2\|u\|_{\infty}$ and

$$
\liminf _{k \rightarrow \infty} \int_{A} g\left(\operatorname{det} D v_{k}\right) \leq \liminf _{k \rightarrow \infty} \int_{A} g\left(\operatorname{det} D u_{k}\right)
$$

for any open set $A \subset \Omega$.

Proof. Up to a subsequence we can suppose that $u_{k} \rightarrow u$ almost everywhere. Let $v_{k}=\pi^{N}\left(u_{k}\right)$ where

$$
\pi^{N}(x)= \begin{cases}x & \text { if }|x| \leq N \\ N \frac{x}{|x|} & \text { otherwise }\end{cases}
$$

with $N=2\|u\|_{\infty}$. Clearly $v_{k} \in W_{\text {loc }}^{1, n}, v_{k} \rightarrow u$ in $W^{1, p}$ and $\left\|v_{k}\right\|_{\infty} \leq 2\|u\|_{\infty}$. To verify the last assertion notice that

and hence

$$
\operatorname{det} D v_{k}(x)=\operatorname{det} D u_{k}(x) 1_{\left\{\left|u_{k}\right| \leq N\right\}}(x) \quad \text { a.e. }
$$

$$
\begin{aligned}
\liminf _{k \rightarrow \infty} \int_{A} g\left(\operatorname{det} D v_{k}\right) & \leq \liminf _{k \rightarrow \infty} \int_{A} g\left(\operatorname{det} D u_{k}\right)+\limsup _{k \rightarrow \infty} g(0)\left|\left\{\left|u_{k}\right|>N\right\} \cap A\right| \\
& =\liminf _{k \rightarrow \infty} \int_{A} g\left(\operatorname{det} D u_{k}\right) .
\end{aligned}
$$

Lemma A.2. Let $u: B\left(x_{0}, R\right) \rightarrow \mathbb{R}^{n}$ be a smooth map and consider $v$ defined by

$$
v(x)=u\left(R \frac{x-x_{0}}{\left|x-x_{0}\right|}+x_{0}\right) .
$$

Then, for $p<n$

$$
\int_{B\left(x_{0}, R\right)}|D v|^{p} \leq C(n, p) R \int_{\partial B\left(x_{0}, R\right)}|D u|^{p} .
$$

Proof. We can suppose $x_{0}=0$ and $R=1$. We have

$$
\begin{aligned}
\int_{B}\left|D\left(u\left(\frac{x}{|x|}\right)\right)\right|^{p} & \leq \int_{B}\left|D u\left(\frac{x}{|x|}\right)\right|^{p} \frac{1}{|x|^{p}} \\
& =\int_{0}^{1} \int_{\partial B}|D u(\omega)|^{p} \mathrm{~d} \mathcal{H}^{n-1}(\omega) \varrho^{n-p-1} \mathrm{~d} \varrho \leq \frac{1}{n-p} \int_{\partial B}|D u(\omega)|^{p} \mathrm{~d} \mathcal{H}^{n-1} .
\end{aligned}
$$

Lemma A.3. Let $u \in C^{1}\left(\bar{Q}\left(x_{0}, R\right)\right)$ and define:

$$
w(x)=u\left(R \frac{x-x_{0}}{\left|x-x_{0}\right|_{\infty}}+x_{0}\right) .
$$


Then, for every $p \geq 1$, we have

$$
\int_{Q_{R}}|u-w|^{p} \leq C(n, p) R^{p} \int_{Q_{R}}|D u|^{p} .
$$

Proof. We can suppose that $x_{0}=0$ and $R=1$. Consider the change of variable:

$$
\left\{\begin{array}{l}
\omega=\frac{x}{|x|_{\infty}} \in \partial Q_{1} \\
\varrho=|x|_{\infty} \in[0,1]
\end{array}\right.
$$

we have

$$
\left|u(x)-u\left(\frac{x}{|x|_{\infty}}\right)\right|^{p}=|u(\varrho \omega)-u(\omega)|^{p} \leq(1-\varrho)^{p-1} \int_{\varrho}^{1}|D u(t \omega)|^{p} \mathrm{~d} t .
$$

Multiplying both sides by $\varrho^{n-1}$ and recalling that $\varrho \leq t$ we obtain

$$
|u(\varrho \omega)-u(\omega)|^{p} \varrho^{n-1} \leq(1-\varrho)^{p-1} \int_{\varrho}^{1}|D u(t \omega)|^{p} t^{n-1} \mathrm{~d} t .
$$

Integrating both members with respect to $\omega \in \partial Q_{1}$ and $\varrho \in[0,1]$ and recalling the co-area formula we have the result.

Acknowledgements. I would like to thank Emanuele Paolini for his invaluable advice, Matteo Focardi and Paolo Marcellini for many useful discussions and suggestions and Francesco Maggi for suggesting me the proof of Lemma A.3. Finally I would like to thank the anonymous referee for her or his suggestions and remarks and for pointing out an error in the proofs of Theorems 6.1 and 6.2 .

\section{REFERENCES}

[1] G. Alberti, S. Baldo and G. Orlandi, Functions with prescribed singularities. J. Eur. Math. Soc. (JEMS) 5 (2003) $275-311$.

[2] L. Ambrosio, N. Fusco and D. Pallara, Functions of bounded variation and free discontinuity problems, Oxford Mathematical Monographs. The Clarendon Press Oxford University Press, New York (2000).

[3] J.M. Ball, Convexity conditions and existence theorems in nonlinear elasticity. Arch. Rational Mech. Anal. 63 (1976) $337-403$.

[4] F. Bethuel, A characterization of maps in $H^{1}\left(B^{3}, S^{2}\right)$ which can be approximated by smooth maps. Ann. Inst. Henri Poincaré Anal. Non Linéaire 7 (1990) 269-286.

[5] F. Bethuel, The approximation problem for Sobolev maps between two manifolds. Acta Math. 167 (1991) 153-206.

[6] G. Bouchitté, I. Fonseca and J. Malý, The effective bulk energy of the relaxed energy of multiple integrals below the growth exponent. Proc. R. Soc. Edinb. Sect. A 128 (1998) 463-479.

[7] H. Brezis and L. Nirenberg, Degree theory and BMO. I. Compact manifolds without boundaries. Selecta Mathematica (N.S.) 1 (1995) 197-263.

[8] H. Brezis and L. Nirenberg, Degree theory and BMO. II. Compact manifolds with boundaries. Selecta Mathematica (N.S.) 2 (1996) 309-368.

[9] H. Brezis, J.-M. Coron and E.H. Lieb, Harmonic maps with defects. Comm. Math. Phys. 107 (1986) 649-705.

[10] R. Coifman, P.-L. Lions, Y. Meyer and S. Semmes, Compensated compactness and Hardy spaces. J. Math. Pures Appl. (9) 72 (1993) 247-286.

[11] S. Conti and C. De Lellis, Some remarks on the theory of elasticity for compressible Neohookean materials. Ann. Scuola Norm. Sup. Pisa Cl. Sci. (5) 2 (2003) 521-549.

[12] B. Dacorogna, Direct methods in the calculus of variations, Applied Mathematical Sciences 78. Springer, New York, second edition (2008).

[13] B. Dacorogna and P. Marcellini, Semicontinuité pour des intégrandes polyconvexes sans continuité des déterminants. $C$. $R$. Acad. Sci. Paris Sér. I Math. 311 (1990) 393-396.

[14] C. De Lellis, Some fine properties of currents and applications to distributional Jacobians. Proc. R. Soc. Edinb. Sect. A 132 (2002) 815-842.

[15] C. De Lellis, Some remarks on the distributional Jacobian. Nonlinear Anal. 53 (2003) 1101-1114.

[16] C. De Lellis and F. Ghiraldin, An extension of Müller's identity Det = det. C. R. Math. Acad. Sci. Paris 348 (2010) 973-976. 
[17] L.C. Evans and R.F. Gariepy, Measure theory and fine properties of functions, Studies in Advanced Mathematics. CRC Press, Boca Raton, FL (1992).

[18] H. Federer, Geometric measure theory, Die Grundlehren der mathematischen Wissenschaften, Band 153. Springer-Verlag New York Inc., New York (1969).

[19] I. Fonseca and W. Gangbo, Degree theory in analysis and applications, Oxford Lecture Series in Mathematics and its Applications. The Clarendon Press Oxford University Press, New York (1995).

[20] I. Fonseca and J. Malý, Relaxation of multiple integrals below the growth exponent. Ann. Inst. Henri Poincaré Anal. Non Linéaire 14 (1997) 309-338.

[21] I. Fonseca and P. Marcellini, Relaxation of multiple integrals in subcritical Sobolev spaces. J. Geom. Anal. 7 (1997) 57-81.

[22] I. Fonseca, N. Fusco and P. Marcellini, On the total variation of the Jacobian. J. Funct. Anal. 207 (2004) 1-32.

[23] I. Fonseca, N. Fusco and P. Marcellini, Topological degree, Jacobian determinants and relaxation. Boll. Unione Mat. Ital. Sez. B Artic. Ric. Mat. (8) 8 (2005) 187-250.

[24] M. Giaquinta, G. Modica and J. Souček, Graphs of finite mass which cannot be approximated in area by smooth graphs. Manuscr. Math. 78 (1993) 259-271.

[25] M. Giaquinta, G. Modica and J. Souček, Remarks on the degree theory. J. Funct. Anal. 125 (1994) $172-200$.

[26] M. Giaquinta, G. Modica and J. Souček, Cartesian currents in the calculus of variations I, Cartesian currents. Springer-Verlag, Berlin (1998).

[27] A. Hatcher, Algebraic topology. Cambridge University Press, Cambridge (2002).

[28] R.L. Jerrard and H.M. Soner, Functions of bounded higher variation. Indiana Univ. Math. J. 51 (2002) 645-677.

[29] J. Malý, L ${ }^{p}$-approximation of Jacobians. Comment. Math. Univ. Carolin. 32 (1991) 659-666.

[30] P. Marcellini, Approximation of quasiconvex functions, and lower semicontinuity of multiple integrals. Manuscr. Math. 51 (1985) 1-28.

[31] P. Marcellini, On the definition and the lower semicontinuity of certain quasiconvex integrals. Ann. Inst. Henri Poincaré Anal. Non Linéaire 3 (1986) 391-409.

[32] P. Marcellini, The stored-energy for some discontinuous deformations in nonlinear elasticity, in Partial differential equations and the calculus of variations II, Progr. Nonlinear Differential Equations Appl. 2, Birkhäuser Boston, Boston, MA (1989) $767-786$.

[33] D. Mucci, Remarks on the total variation of the Jacobian. NoDEA Nonlinear Differential Equations Appl. 13 (2006) $223-233$.

[34] D. Mucci, A variational problem involving the distributional determinant. Riv. Mat. Univ. Parma (to appear).

[35] S. Müller, Higher integrability of determinants and weak convergence in $L^{1}$. J. Reine Angew. Math. 412 (1990) $20-34$.

[36] S. Müller, Det $=$ det. A remark on the distributional determinant. C. R. Acad. Sci. Paris Sér. I Math. 311 (1990) 13-17.

[37] S. Müller, On the singular support of the distributional determinant. Ann. Inst. Henri Poincaré Anal. Non Linéaire 10 (1993) $657-696$.

[38] S. Müller and S.J. Spector, An existence theory for nonlinear elasticity that allows for cavitation. Arch. Rational Mech. Anal. 131 (1995) 1-66.

[39] S. Müller, Q. Tang and B.S. Yan, On a new class of elastic deformations not allowing for cavitation. Ann. Inst. Henri Poincaré Anal. Non Linéaire 11 (1994) 217-243.

[40] S. Müller, S.J. Spector and Q. Tang, Invertibility and a topological property of Sobolev maps. SIAM J. Math. Anal. 27 (1996) 959-976.

[41] E. Paolini, On the relaxed total variation of singular maps. Manuscr. Math. 111 (2003) $499-512$.

[42] A.C. Ponce and J. Van Schaftingen, Closure of smooth maps in $W^{1, p}\left(B^{3} ; S^{2}\right)$. Differential Integral Equations 22 (2009) $881-900$.

[43] T. Schmidt, Regularity of Relaxed Minimizers of Quasiconvex Variational Integrals with (p, q)-growth. Arch. Rational Mech. Anal. 193 (2009) 311-337.

[44] B. White, Existence of least-area mappings of $N$-dimensional domains. Ann. Math. (2) 118 (1983) $179-185$. 